\title{
Mathematical Modeling and Experimental Identification of an Unmanned Helicopter Robot with Flybar Dynamics
}

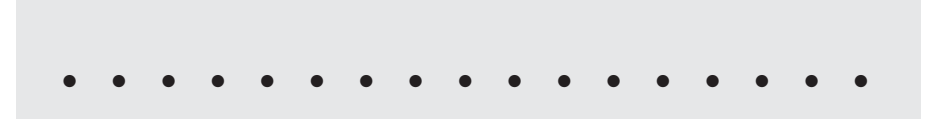

\author{
S. K. Kim and D. M. Tilbury* \\ Department of Mechanical Engineering \\ University of Michigan \\ 2250 G.G. Brown, 2350 Hayward St. \\ Ann Arbor, MI 48109-2125 \\ e-mail: tilbury@umich.edu \\ Received 8 February 2001; accepted 10 November 2003
}

\begin{abstract}
This paper presents a mathematical model for a model-scale unmanned helicopter robot, with emphasis on the dynamics of the flybar. The interaction between the flybar and the main rotor blade is explained in detail; it is shown how the flapping of the flybar increases the stability of the helicopter robot as well as assists in its actuation. The model helicopter has a fast time-domain response due to its small size, and is inherently unstable. Therefore, most commercially available model helicopters use the flybar to augment stability and make it easier for a pilot to fly. Working from first principles and basic aerodynamics, the equations of motion for full six degree-of-freedom with flybar-degree of freedom are derived. System identification experiments and results are presented to verify the mathematical model structure and to identify model parameters such as inertias and aerodynamic constants. (c) 2004 Wiley Periodicals, Inc.
\end{abstract}

\section{INTRODUCTION}

There has been a great deal of interest in the dynamics of unmanned helicopter robots since the last decade, as such unmanned aerial vehicles (UAVs) are being rediscovered. They are an excellent cost-effective and safe way to replace human operators/pilots in mili-

\footnotetext{
*To whom all correspondence should be addressed. Contract sponsor: Michigan Space Grant Consortium Contract sponsor: Rackham Graduate School

Contract grant sponsor: Natural Science Foundation Contract grant numbers: IRI 95-28115 and CMS 98-76039
}

tary, civilian, and commercial areas when there exist significant threats to human lives, or when the environment is not suitable for large human-carrying vehicles. However, helicopter robots are well known to be inherently unstable due to their small size. Over the years, improvements in the design led to the use of an aerodynamically damped gyroscopic device called the flybar, which has become an almost mandatory stability augmentation device for most modern model-scale helicopter robots.

The main contribution of this paper is the detailed modeling of the flybar with the main rotor 
blade and the fuselage movement. This paper also describes the mathematical model that we have derived specifically for a helicopter robot, as well as the system identification experiments we have conducted. The model is based on a rigid-body description of the helicopter, with four actuation inputs representing the four stick positions available to the remotecontrol pilot. Once the mathematical model has been developed, system identification experiments are performed both to verify the structure of the model and to identify some of the unknown model parameters, such as inertias and aerodynamic coefficients.

The outline of the paper is as follows. First, we briefly review some previous work on helicopter modeling. In Section 3, we describe the flybar mathematical model that we have derived, working from first principles and basic aerodynamics. Section 4 describes the system identification technique for identifying the uncertain parameters and presents our results. The paper concludes with a comparison between our nonlinear model and the linear model from ref. 1 and a short summary.

\section{RELATED WORK}

Although there is an extensive literature dealing with the model-scale and full-scale helicopter modeling, direct mathematical modeling of the flybar has been sketchy at best. Researchers have devised linear ${ }^{1,2}$ and nonlinear ${ }^{3,4}$ mathematical models of model helicopters based on the full-scale helicopter models, but with little emphasis on the flybar. System identification ${ }^{1,5-7}$ has also been applied frequently to a linear, parametrized model to avoid the complexity involved with detailed modeling, although all of these ID results are only valid near the nominal hover condition. With most identification works, it is difficult to correlate the identified numerical parameters with the physical parameters, so the understanding of the flybar dynamics still remains incomplete.

Extensive linear ${ }^{8,9}$ and nonlinear ${ }^{10-16}$ modeling and identification ${ }^{17,18}$ works for full-scale helicopters are also available, but most papers are primarily concerned with the aero-elastic flapping rotor mechanism with the rotor hinges modeled as a spring. This is not the primary stabilization method employed by the model-scale helicopter robots; the rotor hub is hingeless and the main blades are too stiff to flap. The flybar dynamics are the dominant stabilization force.

Although some researchers do mention the existence of the flybar used for full-scale ${ }^{19}$ and modelscale helicopters, ${ }^{1,20}$ the authors have been unable to find any recent papers which explain the exact nature of the flybar and its effect on the stability of a model helicopter in a concrete mathematical way. This mechanism is typically discussed by intuition, experience, or a linear numerical identification is used without any explanation of the fundamental dynamics. The main result of this paper is to present this first-principles modeling which has been lacking in the existing literature. At best, Mettler et al. ${ }^{1}$ provide a simple decoupled flybar dynamic model, though based on an incorrect assumption that the flybar's restoring forces are entirely centrifugal. Bramwell ${ }^{21}$ shows a simple uni-directional flybar dynamic expression for full-scale helicopters without any derivation.

\section{MATHEMATICAL MODEL}

Many full scale helicopters have a hinge, either freeflapping or spring-mounted, on the rotor blades, so that the plane of the rotor can be tilted with respect to the helicopter. Such a hinge system allows the rotor blades to flap, increasing the stability. Even helicopters with hingeless rotors still have a significant amount of rotor flapping action, giving a similar kind of stability. However, this flapping behavior increases the time needed for the helicopter to respond to control inputs, and this is the main reason why the flapping mechanism is not used as a stability augmentation device for most small model-scale helicopters.

Most helicopter robots with a rotor span under $1.5 \mathrm{~m}$ have a hingeless, stiff rotor hub design, which forces the position of the fuselage to remain almost fixed with respect to the rotor disc. This results in faster response times, and gives the remote pilot a better sense of motion of the helicopter. However, since the time-domain response of a model helicopter is much faster due to its small size, without any extra stability augmentation devices, a human pilot would have difficulty controlling it. In almost all model helicopters, a large control gyro with an airfoil, referred to as a flybar, is used to improve the stability characteristic around the pitch and roll axes and to minimize the actuator force required. In addition, an electronic gyro is used on the tail rotor to further stabilize the yaw axes.

In this section, we derive the dynamic equations of motion for the model helicopter including its actuator dynamics, with detailed emphasis on the flybar dynamics. A Full 6-DOF helicopter model is also included for completeness. The equations are based on rigid body dynamics, ${ }^{22}$ as well as basic 


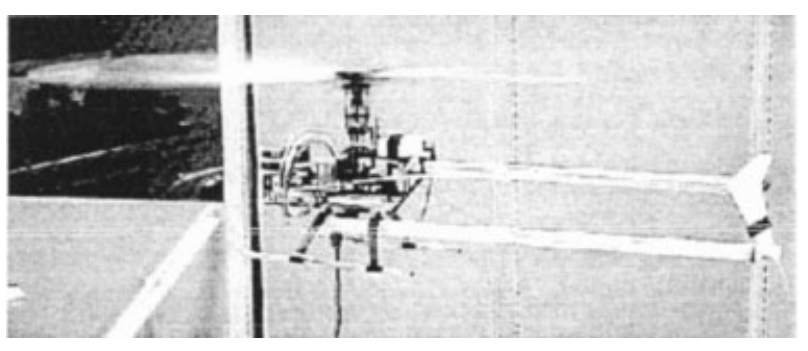

Figure 1. A photograph of the Ikarus ECO electric helicopter used in our experiments (ref. 25). The sensor receiver is attached to the tail to minimize the electromagnetic interference from the motor. The sensor transmitter is fixed to the ground, although different locations were chosen to minimize the distance between the transmitter and receiver depending on the nature of experiment. Because the sensor arrangement already requires the helicopter to be tethered, we power the helicopter motor using a $12 \mathrm{~V}$ car battery.

aerodynamics and helicopter theory. ${ }^{23,24}$ Once the structure of the model has been determined, system identification techniques will be used to validate the model and determine the unknown model parameters.

As shown in Figure 1, our modeling is based on a commercially available model helicopter called Ikarus; ${ }^{25}$ most other brands of model helicopters have a similar structure. The rotor hub is hingeless with a pair of small o-rings supporting the two main blades. It has a $1.02 \mathrm{~m}$ rotor span and weighs $1.36 \mathrm{~kg}$ without the onboard battery. A sensor is attached to the tail fin to measure the position and orientation of the helicopter with respect to an inertial frame. Figure 2 shows some of the onboard components, including a radio receiver which receives command inputs from the remote control box, a motor with a motor controller, four different servos (pitch and roll cyclic, collective pitch, and yaw servo), and a gyro sensor. The single electric motor drives the main and tail rotors simultaneously via gear trains and a timing belt.

\subsection{Coordinates and Frames}

As shown in Figure 3, there are two frames that we consider: the reference inertial frame and the body frame attached to the helicopter. The transformation between the two frames is given by the homogeneous transformation matrix

$$
g_{I B}=\left[\begin{array}{cccc} 
& & & x_{I} \\
& R_{I B} & & y_{I} \\
& & & z_{I} \\
0 & 0 & 0 & 1
\end{array}\right],
$$

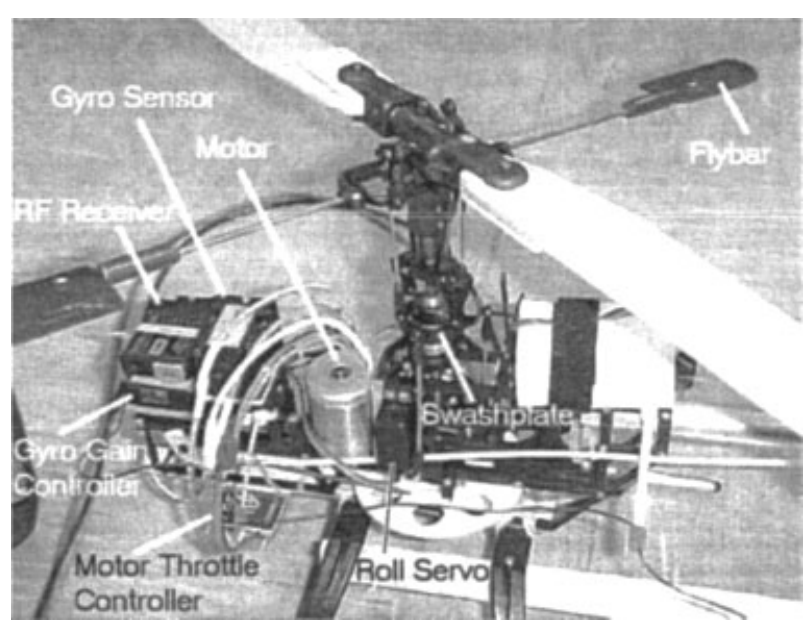

Figure 2. Some of the mechanical and electrical components on the helicopter. A minor reduction in sensor noise was obtained using a brushless motor (ref. 26). All other components are generic model helicopter components.

where the rotation matrix $R_{I B}$ represents the relative orientation between the two frames. The rotation matrix can be expressed in coordinates using yaw-pitchroll $(Z Y X)$ Euler angles as

$$
\begin{aligned}
R_{I B}= & e^{(\hat{\mathbf{z}} \times) \psi} e^{(\hat{\mathbf{y}} \times) \theta} e^{(\hat{\mathbf{x}} \times) \phi} \\
= & {\left[\begin{array}{ccc}
\cos \psi & -\sin \psi & 0 \\
\sin \psi & \cos \psi & 0 \\
0 & 0 & 1
\end{array}\right] \cdot\left[\begin{array}{ccc}
\cos \theta & 0 & \sin \theta \\
0 & 1 & 0 \\
-\sin \theta & 0 & \cos \theta
\end{array}\right] } \\
& \cdot\left[\begin{array}{ccc}
1 & 0 & 0 \\
0 & \cos \phi & -\sin \phi \\
0 & \sin \phi & \cos \phi
\end{array}\right] .
\end{aligned}
$$

Although this representation is singular at $\theta= \pm \pi / 2$, we do not expect to operate the helicopter in that orientation (pointing straight up or down). Thus, we will do most of our derivation in coordinates, where $\left(x_{I}, y_{I}, z_{I}\right)$ are the coordinates of the position of the helicopter with respect to the inertial frame and $\phi, \theta, \psi$ are the Euler angles of the helicopter with respect to the inertial frame.

There are several different velocities that will be used to write the dynamic equations. The linear velocity of the helicopter with respect to the inertial frame is $v_{I}=\left[\begin{array}{lll}\dot{x}_{I} & \dot{y}_{I} & \dot{z}_{I}\end{array}\right]^{T}$. This velocity can also be expressed in the body frame,

$$
v_{B}=R_{I B}^{T} v_{I}
$$




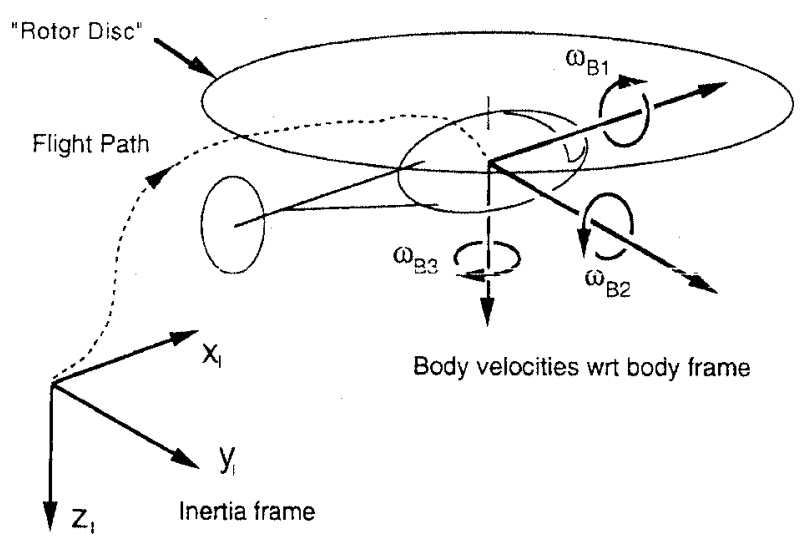

Figure 3. The coordinates defined. The inertial frame is fixed to the ground, and the body frame is fixed to the helicopter. The relative rotation between the two frames is given by the rotation matrix $R_{I B}$, or equivalently by the ZYX Euler angles $(\phi, \theta, \psi)$, and the relative displacement is given by the vector $\left[x_{I} y_{I} z_{I}\right]^{T}$. The rotor disc is an imaginary plane drawn by the tip of main rotor blades.

The angular velocity of the helicopter will also be needed. The angular velocity of the helicopter with respect to the inertial frame, expressed in the body frame, is denoted $\omega_{B}$ and can be computed as

$$
\begin{gathered}
\omega_{B} \times=R_{I B}^{T} \dot{R}_{I B} \\
\omega_{B}=\left[\begin{array}{c}
\dot{\phi}-\dot{\psi} \sin \theta \\
\dot{\theta} \cos \phi+\dot{\psi} \cos \theta \sin \phi \\
-\dot{\theta} \sin \phi+\dot{\psi} \cos \theta \cos \phi
\end{array}\right] \\
=\left[\begin{array}{ccc}
1 & 0 & -\sin \theta \\
0 & \cos \phi & \cos \theta \sin \phi \\
0 & -\sin \phi & \cos \theta \cos \phi
\end{array}\right]\left[\begin{array}{c}
\dot{\phi} \\
\dot{\theta} \\
\dot{\psi}
\end{array}\right] .
\end{gathered}
$$

\subsection{Rigid Body Equations}

We will model the helicopter as a rigid body moving in space, ignoring the effects of the spinning rotor. Although this is not physically true, this assumption helps us to collect all the various inertia effects such as main and tail rotor dynamics into a simple constant mass-inertia matrix. This assumption will be justified through the system identification in Section 4 . The mass of the helicopter is given by $m$, and the fuselage inertias are $I_{x x}, I_{y y}, I_{z z}$. The inertia matrix $\mathcal{I}$, in body coordinates, is

$$
\mathcal{I}=\left(\begin{array}{ccc}
I_{x x} & 0 & -I_{x z} \\
0 & I_{y y} & 0 \\
-I_{x z} & 0 & I_{z z}
\end{array}\right)
$$

Terms such as $I_{x y}$ and $I_{y z}$ are zero due to the symmetry of the helicopter with respect to the $x_{B}-z_{B}$ plane. Although $I_{x z}$ is nonzero, because the helicopter is not symmetric with respect to the $x_{B}-y_{B}$ plane, it is typically much smaller than the other terms. We will ignore it in our model.

The dynamic equations of the helicopter's motion are significantly simplified by expressing them in body coordinates. In these coordinates, Euler's equations for the rigid body motion of the helicopter become $^{22}$

$$
\left[\begin{array}{cc}
m I_{3 \times 3} & 0 \\
0 & \mathcal{I}
\end{array}\right]\left[\begin{array}{c}
\dot{v}_{B} \\
\dot{\omega}_{B}
\end{array}\right]+\left[\begin{array}{c}
\omega_{B} \times m v_{B} \\
\omega_{B} \times \mathcal{I} \omega_{B}
\end{array}\right]=\left[\begin{array}{c}
f_{B} \\
\tau_{B}
\end{array}\right]
$$

The external forces and torques expressed in the body frame are $f_{B}$ and $\tau_{B}$. The body force $f_{B}$ includes aerodynamic drag terms $D_{F}$, the main and tail rotor thrusts $T$ and $T_{T}$, and the gravitational force $m g$ :

$$
f_{B}=\left[\begin{array}{c}
-D_{F_{x}} \\
-D_{F_{y}}-T_{T} \\
-D_{F_{z}}-T
\end{array}\right]+R_{I B}^{T}\left[\begin{array}{c}
0 \\
0 \\
m g
\end{array}\right] .
$$

Torques include the three main directional torques $M_{\phi}, M_{\theta}, M_{\psi}$ as well as a torque due to the offset of the rotor hinge $T \ell_{r}$, and the motor torque $\tau_{m}$ :

$$
\tau_{B}=\left[\begin{array}{c}
M_{\phi} \\
M_{\theta}-T \ell_{r} \\
M_{\psi}+\tau_{m}
\end{array}\right] .
$$

The longitudinal offset between the rotor axis and the helicopter's center of gravity is $\ell_{r}$. Usually this offset is expected to be small for better handling quality. Nevertheless, we will assume this quantity is nonzero for generality. The gravitational acceleration constant is $g$. It is assumed that the helicopter's center of gravity is in-line with the rotor axis laterally, because most components such as motor and gear trains are located along the $x_{B}-z_{B}$ plane, with $y_{B}$ near zero.

The four independent inputs to this model are $T$, the net thrust generated by the rotor, and $M_{\phi}, M_{\theta}, M_{\psi}$, the net moments acting on the helicopter. These four inputs are physically controlled by two joysticks on the radio transmitter, each with two degrees of freedom. The left joystick commands throttle with collective pitch (up/down) and yaw (left/right), and the right joystick commands pitch cyclic (up/ down) and roll cyclic (left/right). This is the most popular configuration used in the U.S. (type 
$\mathrm{II}^{1}$ ). The four values representing the positions of the sticks are encoded in a pulse-width modulated (PWM) signal, and sent via radio link to the helicopter.

To match with our experimental measurements, we will also consider the transformation of the Newton-Euler equation (2) into a new set of coordinates, $q$, which includes the inertial position and velocity of the helicopter, the Euler angles, and the body angular velocity: ${ }^{27}$

$$
q=\left[\begin{array}{llllllllllll}
x_{I} & y_{I} & z_{I} & \dot{x}_{I} & \dot{y}_{I} & \dot{z}_{I} & \phi & \theta & \psi & \omega_{B 1} & \omega_{B 2} & \omega_{B 3}
\end{array}\right]^{T} .
$$

The transformed equations become

$$
\dot{q}=\left[\begin{array}{c}
v_{I} \\
\frac{1}{m} R_{I B} f_{B} \\
\Upsilon \omega_{B} \\
\mathcal{I}^{-1}\left(\tau_{B}-\omega_{B} \times \mathcal{I}_{\omega_{B}}\right)
\end{array}\right]
$$

where $Y$ is the inverse of the matrix shown in (1):

$$
\begin{aligned}
Y & =\left[\begin{array}{ccc}
1 & 0 & -\sin \theta \\
0 & \cos \phi & \cos \theta \sin \phi \\
0 & -\sin \phi & \cos \theta \cos \phi
\end{array}\right]^{-1} \\
& =\left[\begin{array}{ccc}
1 & \sin \phi \tan \theta & \cos \phi \tan \theta \\
0 & \cos \phi & -\sin \phi \\
0 & \sin \phi \sec \theta & \cos \phi \sec \theta
\end{array}\right] .
\end{aligned}
$$

Note that this expression highlights the singularity of the representation at $\theta= \pm \pi / 2$.

\subsection{Aerodynamic Modeling}

Before developing the actuation equations, we introduce some basic aerodynamic terms that will be required. We will assume the rotor system is completely rigid (there is no aeroelasticity effect), and that the airfoil is symmetric and nontwisted. The aerodynamic interaction between the rotor and the fuselage is neglected. The aerodynamic expressions are based on 2-D analysis. This type of modeling is often called level-1 modeling and is appropriate for low bandwidth control and to observe the parametric trends for flying qualities and performance studies. ${ }^{24}$

\footnotetext{
${ }^{1}$ In some countries, people prefer the yaw and the roll controls switched (type I).
}

The rotor span, $R$, is assumed to be the distance between the rotor axis and the rotor tip. We neglect the effect due to "root cutout," where there is no rotor blade at the hub area. ${ }^{23}$ The model helicopter's rotor blade remains nearly rigid due to its short length (0.4 to $0.8 \mathrm{~m}$ ), high rotor speed (1200 to $1900 \mathrm{rpm})$, and hingeless hub design; we assume the rotor is perfectly rigid with no twist.

The descent ratio, $\nu$, represents the air speed component perpendicular to the rotor disc. ${ }^{28}$ To define it, we first need to find the velocity of the hub point with respect to the inertial coordinate frame represented in body coordinates; we denote this velocity by $v_{q}^{b}$. The coordinate of the rotor hub point in the body frame is $q_{b}=\left[\begin{array}{lll}\ell_{r} & 0 & -h_{r}\end{array}\right]^{T}$. The constants $-h_{r}$ and $\ell_{r}$ are the offsets of the rotor from the helicopter's center of gravity in the $x_{B}$ and $z_{B}$ directions, respectively; the rotor hub is in-line with the center of gravity in the $y_{B}$ direction:

$$
v_{q}^{b}=\omega_{B} \times q_{b}+v_{B} .
$$

The descent ratio, $\nu$, which is the airspeed component perpendicular to the rotor disc, can be found as the magnitude of the third element of $v_{q}^{b}$, nondimensionalized by $R \Omega$ :

$$
\nu=\frac{1}{R \Omega} v_{q^{3}}^{b}
$$

When the helicopter is hovering, this ratio will be very small and may be ignored. The inflow ratio, $\lambda$, is the net value of the descent ratio $\nu$ and the induced air velocity. The velocity of the air through the rotor blade $v_{i}$ is defined to be positive when the flow is along the $z_{B}$-axis and is nondimensionalized by $R \Omega$. We choose not to model $\lambda$ in any more detail in this paper due to its inherent complexity: $:^{23}$

$$
\lambda=-\nu+\frac{v_{i}}{R \Omega} .
$$

The most important parameter in determining the lift force is the angle of attack of a blade, which is the angle between the centerline of the blade and the blade velocity vector with respect to the inertial frame. The angle of incidence, which is the angle between the centerline of the blade and the reference frame (such as the rotor axis), is not related to the aerodynamic effects. The lift curve slope, $a$, is the 
slope of the function of the lift force versus angle of attack of the main rotor blade; ${ }^{29}$ we assume here that $a$ is a constant.

\subsection{Flybar Dynamics}

The dynamics of the rotor and the flybar are the most significant elements in the creation of the forces and moments on the helicopter. The actuator dynamics include as states the flapping angle and velocity $(\beta, \dot{\beta})$ of the flybar and the position and angular velocity $(\xi, \Omega)$ of the main rotor blade. As mentioned before, the flybar plays a major role in augmenting the stability of a model helicopter. The flybar mechanism is sketched in Figure 4. The original concept of this design came from full-scale helicopter stabilization devices first used in the 1950s. The Bell stabilizing system had a bar with weights at each end, and the flapping motion of the bar was governed by a separate damper. The Hiller system replaced the damper and the weights with an airfoil. During the early 1970s, the design was simplified and improved for model-scale helicopters. ${ }^{30}$ This system is often called a Bell-Hiller mixer, because it incorporates some of design aspects of both Bell and Hiller designs. The fundamental concept for the model helicopter's rotor hub design is identical to the Hiller system, although the mechanical detail resembles the Bell system. Since the early 1970s, the design of the model helicopter's rotor hub has gone through many changes and improvements; it reached its current state in the early 1990s. The rotor hub design presented in Figure 4, hingeless with Bell-Hiller mixer, represents currently the most popular and widely accepted design as the best compromise between performance and stability. However, it is suited more toward aerobatic maneuvers than smooth near-hover maneuvers that do not require large and fast pitch or roll movements. Stability can be increased if the Bell input is removed and/or the main blade is allowed to flap, but the helicopter would then respond more slowly. ${ }^{30}$

Figure 4 shows the two arms coming from the swashplate. One arm, marked Bell input, allows the blade pitch to be changed directly from the cyclic servo actuator. The response with respect to this arm alone is fast, but lacks stability. Meanwhile, the other arm, marked Hiller input, allows the pitch of the flybar to be changed. The flybar then flaps, and this flapping motion causes the pitch of the main blade to change. The pilot's cyclic control input $\delta_{\theta}$ and $\delta_{\phi}$ are the displacements of the lower swashplate as per Figure 4 . There is a direct relationship between the cyclic input applied to the main blades $\delta_{\text {cyc }}$ (which is a func-

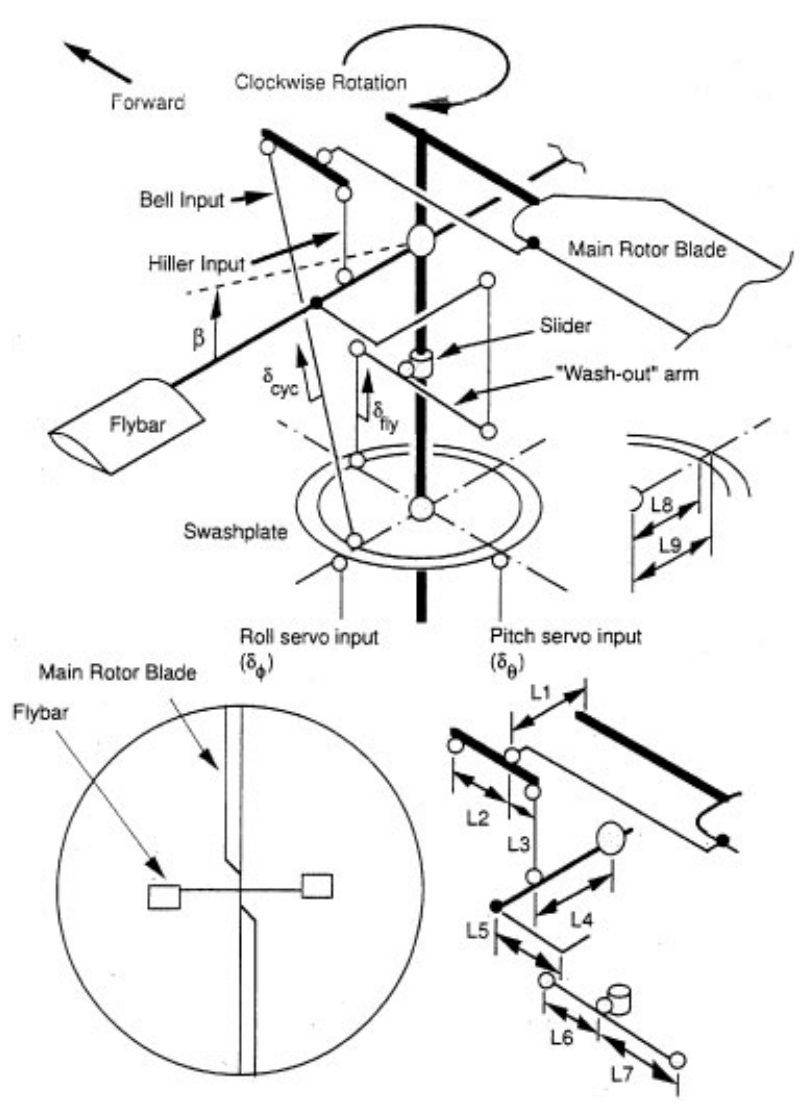

Figure 4. The basic structure of the model helicopter's cyclic/collective control system. Only one side of the assembly is shown for clarity; the other is simply a mirror image. The flapping angle of the flybar, $\beta$, is the angle of the flybar with respect to the body coordinate frame attached to the rotor hub. It is zero when the flybar is perpendicular to the rotor axis. Ball joints are shown as " $\bigcirc$," and fixed joints are shown as "O." The cyclic pitch input to the main rotor blade is controlled by the combination of the Bell input from the swashplate and the Hiller input from the flybar. The collective pitch input to the main rotor blade is controlled by raising or lowering the swashplate. The "wash-out" arm with the slider prevents the collective input from affecting the flybar. The flybar angle of incidence and the flapping angle are thus separated from the collective input. The slider slides only when the collective input is applied. The slider remains stationary when only a cyclic input is applied.

tion of $\delta_{\theta}$ and $\delta_{\phi}$ ) and the cyclic angle of the rotor blades $\theta_{\text {cyc }}$. A similar relationship exists between the cyclic input applied to the flybar $\delta_{\text {fly }}$ and the flapping angle of the flybar $\beta$. The orientation of the main blade with respect to the helicopter is given by $\xi$. The factor $L_{8} / L_{9}$ reflects the different diameters of the upper and lower swashplates. Note the $90^{\circ}$ phase difference between $\delta_{\text {cyc }}$ and $\delta_{f l y}$, due to the geometry of the rotor/flybar assembly shown in Figure 4: 


$$
\begin{gathered}
\delta_{\text {cyc }}(\xi)=-\frac{L_{8}}{L_{9}}\left(\delta_{\theta} \sin \xi+\delta_{\phi} \cos \xi\right), \\
\delta_{\mathrm{fly}}(\xi)=-\frac{L_{8}}{L_{9}}\left(\delta_{\theta} \cos \xi-\delta_{\phi} \sin \xi\right) .
\end{gathered}
$$

An important assumption at this point is that the rotor system does not apply reaction forces back to the actuators, including the flybar (the flybar is considered to be another actuator to the main blades). This is equivalent to assuming the actuators are able to apply infinite amount of forces to the airfoils. This assumption is reasonable because the airfoil of the main blades is symmetric and the blades are hinged along the center of lift. Ideally, the moment required to rotate the blade at this point should be very small. ${ }^{29}$

When a cyclic input is applied by the pilot, the flybar creates lift which tilts the flybar disc. The flybar acts not only as a main blade angle actuator but also as a stabilizer. If the cyclic input were applied to the main blades only, large control forces on the cyclic servo actuators would be required. ${ }^{31}$ By applying the cyclic control to the flybar and allowing the flybar to apply a secondary cyclic input to the main blade, the servo load is significantly reduced.

The flybar is hinged on the main axis to flap freely and rotate around the main axis while remaining $90^{\circ}$ out of phase with respect to main blades. Therefore, its yaw angle with respect to the body frame is $\xi^{\prime}=\xi+\pi / 2$. Its flapping (pitching) angle, $\beta$, is measured with respect to the plane perpendicular to the main rotor axis. The roll motion of the flybar changes the angle of attack of the flybar, $\theta_{\text {fly }}$. However, one can safely neglect the contribution of this motion in deriving an expression governing $\beta$, because of the flybar's small inertia. The roll motion of the flybar only affects the lift created by changing the angle of incidence of the main rotor blades. The rotation matrix which relates the flybar position to the body coordinates of the helicopter is denoted $R_{B F}$; that is,

$$
R_{B F}=e^{(\hat{z} \times)\left(\xi^{\prime}\right)} e^{(\hat{y} \times) \beta} e^{(\hat{x} \times) 0} .
$$

As before, the rotation matrix between the helicopter body frame and the inertial frame is $R_{I B}$. The rotation matrix relating the flybar frame to the inertial frame is thus the product of the two: $R_{I F}=R_{I B} R_{B F}$.

The rotational inertia of the flybar $\hat{I}$ is unified as
$I_{f}$; this is a reasonable assumption because most of the flybar mass is concentrated at the tip region. There are $n$ rotor blades (in our helicopter, $n=2$ ):

$$
\begin{aligned}
& I_{f}=n \int_{R_{1}}^{R_{2}} r^{2} m_{F} d r, \\
& \hat{I}=\operatorname{diag}\left[\begin{array}{lll}
0 & I_{f} & I_{f}
\end{array}\right] .
\end{aligned}
$$

The angular velocity of the flybar $\omega_{F}$ involves the velocity of the flybar and the helicopter simultaneously; it can be found by computing $R_{I F}^{T} \dot{R}_{I F}:{ }^{22}$

$$
\begin{gathered}
\left(\omega_{F} \times\right)=R_{I F}^{T} \dot{R}_{I F}=R_{B F}^{T}\left(\omega_{B} \times\right) R_{B F}+R_{B F}^{T} \dot{R}_{B F}, \\
\omega_{F}=\left[\begin{array}{c}
-\omega_{B 3} \sin \beta+\cos \beta\left(\omega_{B 1} \cos \xi^{\prime}+\omega_{B 2} \sin \xi^{\prime}\right) \\
\omega_{B 2} \cos \xi^{\prime}-\omega_{B 1} \sin \xi^{\prime} \\
\omega_{B 3} \cos \beta+\sin \beta\left(\omega_{B 1} \cos \xi^{\prime}+\omega_{B 2} \sin \xi^{\prime}\right)
\end{array}\right] \\
+\left[\begin{array}{c}
-\dot{\xi}^{\prime} \sin \beta \\
\dot{\beta} \\
\dot{\xi}^{\prime} \cos \beta
\end{array}\right] .
\end{gathered}
$$

Note that the flybar velocity can be expressed as the contribution of the helicopter motion expressed in the flybar frame and the contribution of the flapping of the flybar. We will assume that the rotor angular velocity is constant; thus, $\dot{\xi}^{\prime}=\dot{\xi}=\Omega$.

The external moment applied to the flybar around the pitch axis is $\tau_{F 2}$; it is mainly created by aerodynamic lift. To find the total torque, we integrate along the length of the flybar. Since we will only be interested in the second of these three equations, we will not consider the first and the third elements in any detail:

$$
\boldsymbol{\tau}_{F}=\left[\begin{array}{llll}
\tau_{F_{1}} & n \int_{R_{1}}^{B R_{2}} r d L & \tau_{F_{3}}
\end{array}\right]^{T} .
$$

The constant tip loss factor $B$ takes into account the fact that a finite length airfoil would lose some of the lift generated due to the wing tip vortex effect. ${ }^{23}$ This effect is applied by "reducing" the length of the blade by the factor of $B$. Tip loss is often set to $B=0.97 .^{23}$

The lift element $d L$ depends on the angular velocity of the flybar and its angle of attack $\theta_{\text {fly }}$. The angle of attack of the flybar will be influenced by the pilot input $\delta_{\text {fly }}$ and the ratio between the second element of the angular velocity vector of the flybar $\omega_{F 2}$ and $\Omega .{ }^{24}$ Correction factors $\alpha_{1}$ and $\alpha_{2}$ have been 
included to compensate for the simplified aerodynamics; they will be identified experimentally in Section 4:

$$
\begin{aligned}
& d L=\frac{1}{2} \rho(\Omega r)^{2} a \theta_{r m} c_{2} d r, \\
& \theta_{\mathrm{fly}}=\frac{\alpha_{1} L_{7}}{L_{5} L_{6}} \delta_{\mathrm{fly}}-\frac{\alpha_{2} \omega_{F 2}}{\Omega} .
\end{aligned}
$$

Once the external force, angular velocity, and inertia have been defined, the motion of the flybar can be described using the Euler equation.

$$
\begin{gathered}
\tau_{F}=\omega_{F} \times \hat{I} \omega_{F}+\hat{I} \dot{\omega}_{F} \\
{\left[\begin{array}{c}
\tau_{F 1} \\
n \int_{R_{1}}^{B R_{2}} r d L \\
\tau_{F 3}
\end{array}\right]=\left[\begin{array}{c}
0 \\
-I_{f} \omega_{F 1} \omega_{F 3} \\
I_{f} \omega_{F 1} \omega_{F 2}
\end{array}\right]+\left[\begin{array}{c}
0 \\
I_{f} \dot{\omega}_{F 2} \\
I_{f} \dot{\omega}_{F 3}
\end{array}\right] .}
\end{gathered}
$$

The second of these three equations describes the flapping motion of the flybar. The small angle approximation is used for $\beta$ since it should not exceed about $\pm 25^{\circ}$. For example, if roll motion only is considered, the second equation of (17) becomes the following:

$$
\begin{gathered}
\ddot{\beta}-\cos \xi \ddot{\phi}+2 \Omega \sin \xi \dot{\phi}-\sin ^{2} \xi \beta \dot{\phi}^{2}+\Omega^{2} \beta \\
=\frac{n \Omega^{2}}{8} \frac{\rho a c_{2}\left(B^{4} R_{2}^{4}-R_{1}^{4}\right)}{I_{f}}\left(\frac{\alpha_{1} L_{7} L_{8}}{L_{5} L_{6} L_{9}} \delta_{\phi} \sin \xi\right. \\
\left.-\frac{\alpha_{2}(\dot{\beta}-\dot{\phi} \cos \xi)}{\Omega}\right) .
\end{gathered}
$$

The above expression is similar to the Bell stabilizing bar equation from a full-scale helicopter. ${ }^{21}$ In the absence of aerodynamic forces and external moments, the flybar behaves as a gyroscope, maintaining its orientation relative to inertial space, ${ }^{23}$ as shown in Figure 5. An external disturbance would upset the helicopter angles $\theta$ and $\phi$, effectively changing $\beta$. This nonzero $\beta$ acts to apply an appropriate compensation input to the main blade cyclic control system to stabilize the helicopter. Figure 6 compares the behavior of the model with and without the flybar.

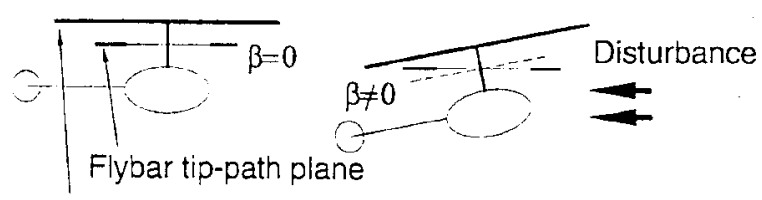

Main blade tip-path plane

Figure 5. The stabilizing effect of the flybar. In a hovering situation, the flybar angle $\beta$ is zero. If a wind gust or other disturbance knocks the helicopter out of its equilibrium, the flybar, which is hinged freely, will continue to rotate in the same inertial plane. Its angle with respect to the main blade becomes nonzero, and it will help bring the helicopter back to equilibrium through its action on the cyclic angle of the main blade.

\subsection{Creation of Forces and Moments}

Once the flybar dynamics have been found, the rest of the actuator dynamics can be derived. The four independent inputs to the rigid body dynamics are the thrust generated by the main rotor blade $T$, the moments created by the main rotor blade around the roll and the pitch axes $\left(M_{\phi}\right.$ and $\left.M_{\theta}\right)$, and the yaw moment $M_{\psi}$.

\subsubsection{Main Rotor Thrust}

The throttle/collective command $\delta_{0}$ controls the thrust to the main motor $(T)$ as well as the collective
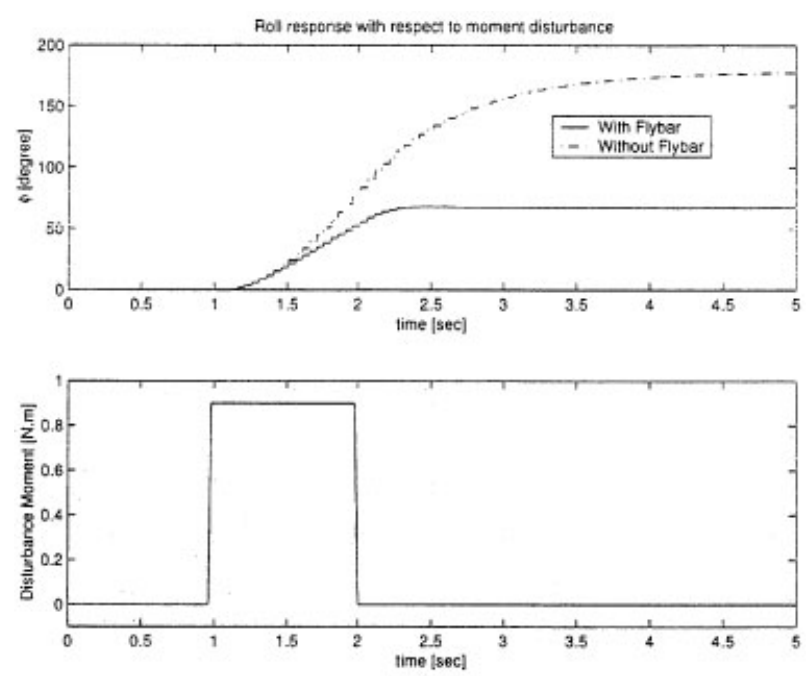

Figure 6. The roll response of the helicopter with respect to the roll moment disturbance is compared by simulation using the analytic model presented in Section 3.6. With the flybar, the response tends to settle within about $0.3 \mathrm{~s}$. Simulating the no-flybar condition by setting $\beta=0$, the helicopter reacts more strongly to the disturbance and takes longer to settle. 
pitch $\left(\theta_{0}\right)$ of the rotor blades. As the blade pitch increases, more lift is created, and the rotational motion of the main rotor blade is converted into vertical thrust. We define $\delta_{o}$ as the collective pitch angle measured when the helicopter is at rest. The mechanical linkages in the collective control system of Figure 4 introduce significant compliance; thus, a secondorder mass-spring-damper model is used to model the actual collective pitch angle $\theta_{o}$ while flying:

$$
\ddot{\theta}_{o}+K_{5} \dot{\theta}_{o}+K_{6} \theta_{o}=K_{4} \cdot \delta_{o}
$$

An adequate torque $\tau_{m}$ linked to $\delta_{o}$ is applied to keep $\Omega$ constant. Sometimes, an electronic throttle governor is used for this purpose, but mostly an empirical curve determining the necessary functional relationship between $\tau_{m}$ and $\delta_{o}$ is programmed into the radio transmitter.

The expression for the rotor thrust $T$ near hover in terms of the collective pitch is obtained from ${ }^{28}$

$$
T=\frac{n}{2} \operatorname{ac} \rho \pi R^{3} \Omega^{2}\left(\frac{B^{3}}{3} \theta_{o}-\frac{B^{2}}{2} \lambda\right) .
$$

This is an expression of the average thrust contribution about one revolution of the rotor blades; the average contribution of the cyclic pitch is zero.

\subsubsection{Pitch and Roll Moments}

The pitch and roll commands vary the cyclic pitch $\left(\theta_{\text {cyc }}\right)$ of the rotor blades around each cycle of rotation, creating different amounts of lift in different regions (as shown in Figure 7). These differing amounts of thrust create a moment around the rotor hub, and can thus create pitch and roll moments on the helicopter.

The moment created by a roll cyclic input is derived by summing the forces around a revolution and along a rotor blade. As per Figure 7, a positive pitch input $\delta_{\theta}$ produces a roll moment $M_{\phi}$, but acts as a pitch moment $M_{\theta}$ due to the precession effect. ${ }^{23}$ For clockwise rotating blades, this effect is taken into account by adding $\pi / 2$ to the sine and cosine functions in the argument of the integral:

$$
\left[\begin{array}{l}
M_{\phi} \\
M_{\theta}
\end{array}\right]=\frac{n}{2 \pi} \int_{0}^{2 \pi} \int_{0}^{B R} r\left[\begin{array}{c}
-\sin \left(\xi+\frac{\pi}{2}\right) \\
\cos \left(\xi+\frac{\pi}{2}\right)
\end{array}\right] d L_{m} d \xi
$$

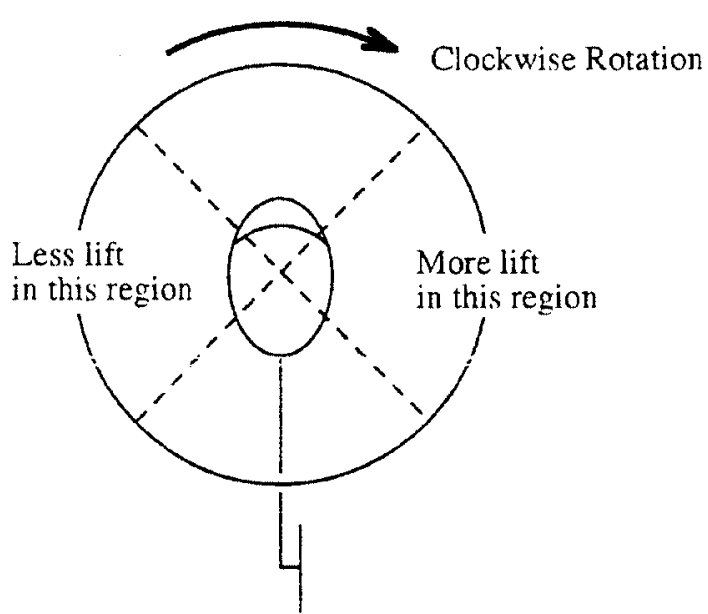

Figure 7. The top view of the helicopter. The lift distribution on the rotor disc when a forward cyclic (pitch forward) input is applied. Viewed from above, when the blade rotation is clockwise and a forward cyclic input is applied, there will be less lift on the left side and more lift on the right side due to the cyclic pitch change. The resulting moment is applied to the main axis $90^{\circ}$ later due to the precession effect. Since the blade spins clockwise, the smaller lift on the left side applies a pitch down moment as a result. The helicopter will then pitch forward.

Note that the above expression represents the average moment created by the rotor blades around a revolution. We assume that the rotor angular velocity is constant; thus $\xi=\Omega t$.

The lift element equation is similar to the flybar lift used in Eq. (14):

$$
d L_{m}=\frac{1}{2} \rho(\Omega r)^{2} a \theta_{\mathrm{cyc}} \mathcal{C} d r
$$

Geometrically, the cyclic pitch angle $\theta_{\text {cyc }}$ is affected by two different kinds of inputs as shown in Figure 4: a direct input by the pilot and an indirect input from the flybar. The geometry of the hub linkages indicate that $\theta_{\text {cyc }}$ will be the weighted sum of these two inputs. Aerodynamically, similar to the flybar, the angle of attack of the main blade will also be influenced by the ratio between the second element of the angular velocity vector of the main blade $\omega_{M 2}$ and $\Omega .^{24}$

To find the angular velocity of the main rotor blade $\omega_{M 2}$, we use a similar derivation method to that of the flybar case. The rotation matrix which relates the main blade position to the body coordinates of the helicopter is denoted $R_{B M}$. Since the main blades do not flap, the pitch angle is zero. Also the roll angle is neglected: 


$$
R_{B M}=e^{(\hat{z} \times)(\xi)} e^{(\hat{y} \times) 0} e^{(\hat{x} \times) 0} .
$$

The rotation matrix relating the main blade frame to the inertial frame is $R_{I M}=R_{I B} R_{B M}$. Similar to the flybar case, correction factors $\alpha_{3}$ and $\alpha_{4}$ were added to compensate for the simplified aerodynamics; they will also be determined in Section 4 .

Therefore,

$$
\begin{gathered}
\left(\omega_{M} \times\right)=R_{I M}^{T} \dot{R}_{I M}=R_{B M}^{T}\left(\omega_{B} \times\right) R_{B M}+R_{B M}^{T} \dot{R}_{B M}, \\
\omega_{M 2}=-\omega_{B 2} \sin \xi-\omega_{B 1} \cos \xi \\
\theta_{\text {cyc }}=\alpha_{3}\left(\frac{L_{3}}{L_{1}\left(L_{2}+L_{3}\right)} \delta_{r m}+\frac{L_{2} L_{4}}{L_{1}\left(L_{2}+L_{3}\right)} \beta\right)-\alpha_{4} \frac{\omega_{M 2}}{\Omega} .
\end{gathered}
$$

The cyclic angle $\theta_{\text {cyc }}$, and hence the pitch and roll moments, depend on the pitch and roll inputs through $\delta_{r m}$ as well as on the flybar flapping angle $\beta$.

\subsubsection{Yaw Moment}

The tail rotor on a helicopter is used to counteract the yaw moment created by the main rotor blades and to give a yaw directional control; thus, altering the amount of pitch on the tail rotor blades can create more or less total yaw moment for the helicopter. The motor yaw torque applied to the helicopter body by the motor is denoted $\tau_{m}$ and is modeled as proportional to the throttle/collective input $\delta_{0}$ :

$$
\tau_{m}=-K_{m} \cdot \delta_{o} .
$$

The yaw command $\delta_{\psi}$ is the collective pitch of the tail rotor blades. There is no cyclic input for the tail rotor blades, only a collective pitch angle. The angular velocity of the tail rotor blades is related to the angular velocity of the main rotor blades through a constant $K_{\Omega}$. This is realized by a simple gear connection or a pulley:

$$
\Omega_{T}=K_{\Omega} \cdot \Omega .
$$

The thrust generated by the tail rotor is found in a similar manner to the thrust of the main rotor (20), replacing with the values from the tail rotor where appropriate:

$$
T_{T}=\frac{n}{2} a_{T} \mathcal{c}_{T} \rho \pi R_{T}^{3} \Omega_{T}^{2}\left(\frac{B^{3}}{3} \delta_{\psi}-\frac{B^{2}}{2} \lambda_{T}\right) .
$$

The subscripts $T$ indicate that the values pertain to the tail rotor. The thrust of the tail rotor times the distance $L_{T}$ between the main and tail rotor axes creates the yaw moment on the helicopter to counteract the spin of the blades.

In addition, there is a damping term in the equation for $M_{\psi}$ due to the presence of an electronic gyro on the model helicopter. A simple linear model $K_{g} \omega_{B 3}$ is used, although more sophisticated (PI controlled) gyros are now available: ${ }^{32}$

$$
M_{\psi}=T_{T} L_{T}-K_{g} \omega_{B 3} .
$$

\subsection{Complete Model}

A block diagram of the complete mathematical model of the helicopter is shown in Figure 8. The inputs $\delta$ are the four stick positions of the model helicopter, and the outputs $q$ are the rigid body positions, orientations, and velocities. Internal variables include the rotor orientation $\xi$, the flybar flapping angle $\beta$, and the collective pitch angle $\theta_{0}$. The following set of differential and integral equations relate the input to output:

$$
\begin{aligned}
& \ddot{\theta}_{o}+K_{5} \dot{\theta}_{o}+K_{6} \theta_{o}=K_{4} \cdot \delta_{o}, \\
& \tau_{m}=-K_{m} \cdot \delta_{0}, \\
& T=\frac{n}{2} \operatorname{ac} \rho \pi R^{3} \Omega^{2}\left(\frac{B^{3}}{3} \theta_{0}-\frac{B^{2}}{2} \lambda\right), \\
& T_{T}=\frac{n}{2} a_{T} \mathcal{c}_{T} \rho \pi R_{T}^{3} \Omega_{T}^{2}\left(\frac{B^{3}}{3} \delta_{\psi}-\frac{B^{2}}{2} \lambda_{T}\right), \\
& q=\left[\begin{array}{llllllllllll}
x_{I} & y_{I} & z_{I} & \dot{x}_{I} & \dot{y}_{I} & \dot{z}_{I} & \phi & \theta & \psi & \omega_{B 1} & \omega_{B 2} & \omega_{B 3}
\end{array}\right]^{T}, \\
& \dot{q}=\left[\begin{array}{c}
v_{I} \\
\frac{1}{m} R_{I B} f_{B} \\
\Upsilon \omega_{B} \\
\mathcal{I}^{-1}\left(\tau_{B}-\omega_{B} \times \mathcal{I} \omega_{B}\right)
\end{array}\right], \\
& f_{B}=\left[\begin{array}{c}
-D_{F_{x}} \\
-D_{F_{y}}-T_{T} \\
-D_{F_{z}}-T
\end{array}\right]+R_{I B}^{T}\left[\begin{array}{c}
0 \\
0 \\
m g
\end{array}\right],
\end{aligned}
$$




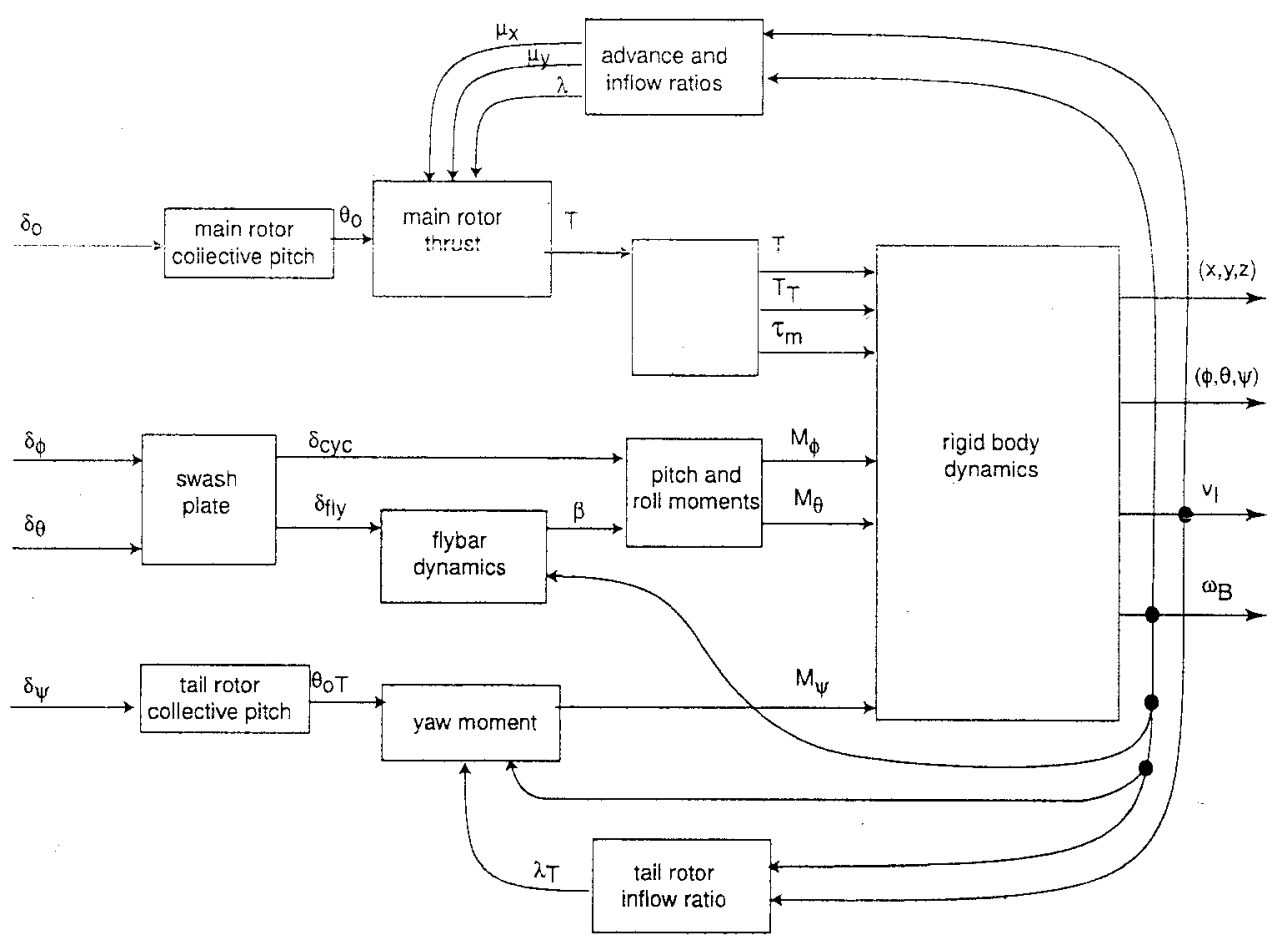

Figure 8. Block diagram of the helicopter model. The pilot inputs are $\delta_{t}, \delta_{\phi}, \delta_{\theta}, \delta_{\psi}$ and the outputs are the position, orientation, and velocity of the helicopter. Internal variables include the rotor orientation $\xi$, the flybar flapping angle $\beta$, and the collective pitch angle $\theta_{0}$.

$$
\begin{gathered}
\tau_{B}=\left[\begin{array}{c}
M_{\phi} \\
M_{\theta}-T \ell_{r} \\
M_{\psi}+\tau_{m}
\end{array}\right], \\
{\left[\begin{array}{l}
M_{\phi} \\
M_{\theta}
\end{array}\right]} \\
=\frac{n a c \rho \Omega^{2}}{4 \pi} \int_{0}^{2 \pi} \int_{0}^{B R}\left[\begin{array}{c}
-\sin \left(\xi+\frac{\pi}{2}\right) \\
\cos \left(\xi+\frac{\pi}{2}\right)
\end{array}\right] r^{3} \theta_{r m} d r d \xi
\end{gathered}
$$

$$
M_{\psi}=T_{T} L_{T}-K_{g} \omega_{B 3},
$$

$$
\delta_{\text {cyc }}(\xi)=-\frac{L_{8}}{L_{9}}\left(\delta_{\theta} \sin \xi+\delta_{\phi} \cos \xi\right)
$$

$$
\delta_{\mathrm{fly}}(\xi)=-\frac{L_{8}}{L_{9}}\left(\delta_{\theta} \cos \xi-\delta_{\phi} \sin \xi\right),
$$

$$
\begin{gathered}
\omega_{F}=\left[\begin{array}{c}
-\omega_{B 3} \sin \beta+\cos \beta\left(\omega_{B 1} \cos \xi^{\prime}+\omega_{B 2} \sin \xi^{\prime}\right) \\
\omega_{B 2} \cos \xi^{\prime}-\omega_{B 1} \sin \xi^{\prime} \\
\omega_{B 3} \cos \beta+\sin \beta\left(\omega_{B 1} \cos \xi^{\prime}+\omega_{B 2} \sin \xi^{\prime}\right)
\end{array}\right] \\
+\left[\begin{array}{c}
-\dot{\xi}^{\prime} \sin \beta \\
\dot{\beta} \\
\dot{\xi}^{\prime} \cos \beta
\end{array}\right], \\
-I_{f} \omega_{F 1} \omega_{F 3}+I_{f} \dot{\omega}_{F 2}=n \int_{R_{1}}^{B R_{2}} \frac{1}{2} \rho(\Omega r)^{2} a\left(\frac{\alpha_{1} L_{7}}{L_{5} L_{6}} \delta_{f l y}\right. \\
\quad-\frac{\alpha_{2} \omega_{F 2}}{\Omega} c_{2} d r \\
\lambda=-\nu+\frac{v_{i}}{R \Omega} \\
\lambda_{T}=-\nu_{T}+\frac{v_{i} T}{R_{T} \Omega} .
\end{gathered}
$$

This set of system equations cannot be easily simulated because the flybar flapping angle $\beta$ is a 

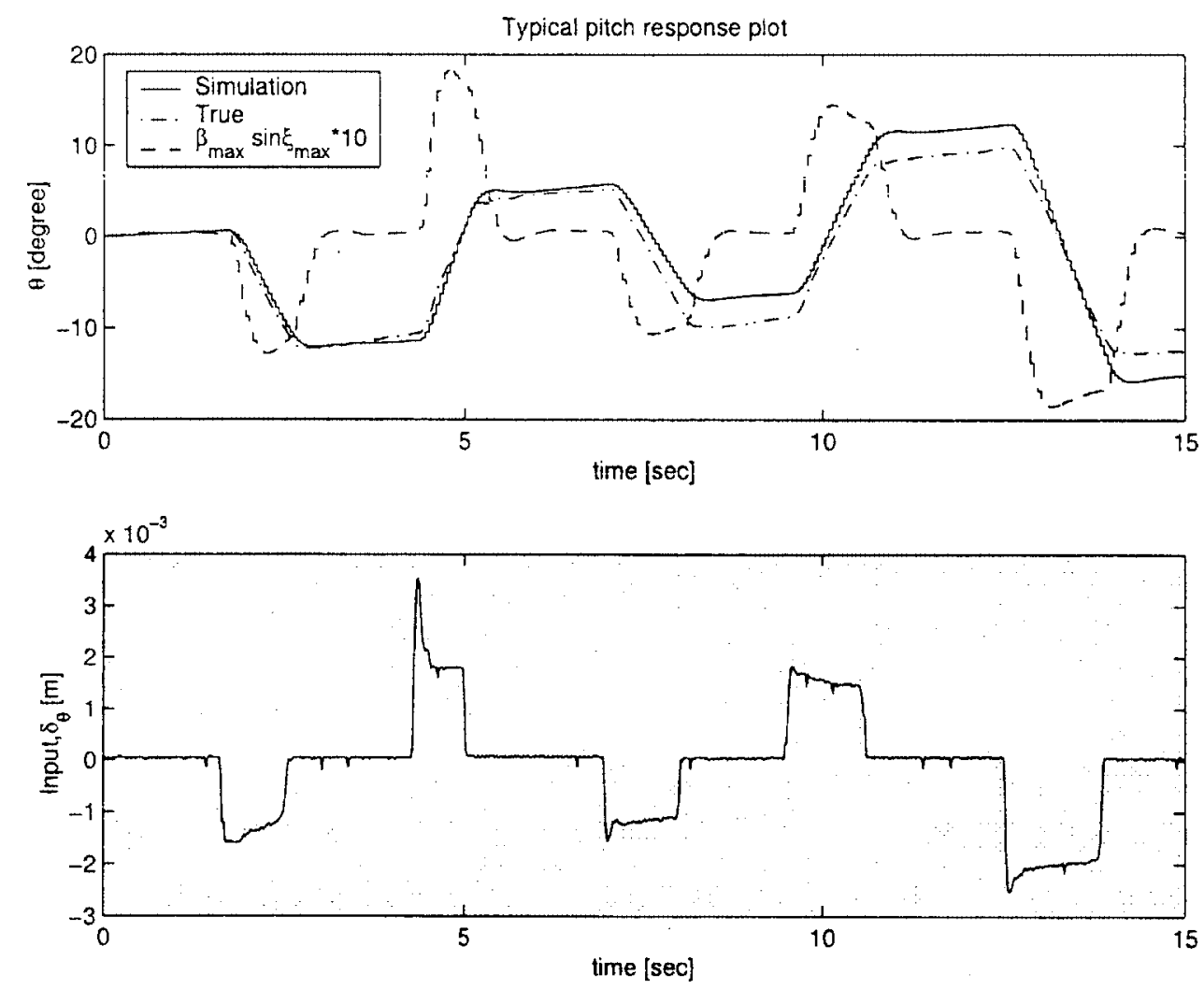

Figure 9. A typical pitch response simulation based on the nonlinear flybar model. Note that the flybar-disk tilt angle, $\beta_{\max } \sin \xi_{\max }$, tends to "subtract" the pilot input by lagging motion. It is magnified by $10 \times$ for clarity.

function of the rotor angle $\xi$, but the roll moment Eq. (21) cannot be solved directly unless $\beta$ is expressed explicitly in terms of $\xi$. For simulation purposes, we approximate the flapping angle $\beta$ as a sinusoidal function around each revolution:

$$
\beta \approx \beta_{\max } \cos \left(\xi-\xi_{\max }\right)
$$

where

$$
\begin{gathered}
\beta_{\max }=\max _{0 \leqslant \xi<2 \pi} \beta(\xi), \\
\xi_{\max }=\arg \max _{0 \leqslant \xi<2 \pi} \beta(\xi) .
\end{gathered}
$$

Using this approximation, the integrals in Eq. (21) can be computed analytically:

$$
\begin{aligned}
M_{\phi}= & \frac{n \rho \Omega^{2} a c R^{4} B^{4}}{16 L_{1}\left(L_{2}+L_{3}\right)}\left(\alpha_{3}\left(\frac{L_{3} L_{8}}{L_{9}} \delta_{\phi}-L_{2} L_{4} \beta_{\max } \cos \xi_{\max }\right)\right. \\
& \left.-\alpha_{4} L_{1}\left(L_{2}+L_{3}\right) \frac{\omega_{B 1}}{\Omega}\right)
\end{aligned}
$$

$$
\begin{aligned}
M_{\theta}= & \frac{n \rho \Omega^{2} a c R^{4} B^{4}}{16 L_{1}\left(L_{2}+L_{3}\right)}\left(\alpha_{3}\left(\frac{L_{3} L_{8}}{L_{9}} \delta_{\theta}-L_{2} L_{4} \beta_{\max } \sin \xi_{\max }\right)\right. \\
& \left.-\alpha_{4} L_{1}\left(L_{2}+L_{3}\right) \frac{\omega_{B 2}}{\Omega}\right) .
\end{aligned}
$$

The system model can thus be reduced to a set of purely differential equations. A Simulink block diagram of the model has been built, and is used to compare the model with data taken as described in Section 4 . In the simulation, the parameters $\beta_{\max }$ and $\xi_{\max }$ are determined for each rotor blade revolution. The $\beta$ and $\omega_{B}$ terms in the $M_{\phi}$ and $M_{\theta}$ pitch and roll moments serve as damping terms in the rigid body equations, increasing the stability of the system from the double-integrator model. Figure 9 shows typically how $\beta_{\max }$ reacts to a pilot input.

In Section 4, we will use this mathematical model, together with experimental data, to estimate the physical parameters of the helicopter model along with the correction factors used in the model. 


\section{SYSTEM IDENTIFICATION}

The main purpose of the system identification is to find physical parameters used in the previous mathematical modeling section. Because of the complexity of the nonlinear model, the system identification is performed on a SISO basis, using specially-built stands to restrict the motion of the helicopter to one degree of freedom. Four different stands were built to isolate the fuselage motion into a set of simple SISO systems. For example, for pitch motion, only the pitch cyclic input was excited. All other inputs including collective, roll cyclic, and tail rotor control were fixed at a near trim input condition. The stand only allowed pitch motion, and therefore only the pitch angle was measured, and the transfer function from the roll cyclic to the pitch motion was identified using standard system identification techniques. Mathematically, the stand makes the roll and yaw inertia nearly infinite as the large inertia of the stand is added to the inertia of the helicopter, effectively preventing the motion in roll and yaw axis.

Going back to the full nonlinear model derived in Section 3, the nonlinear equations were restricted to the SISO case (for example, pitch) and linearized around the hover condition to determine the continuous transfer functions for each degree of freedom. The coefficients of this transfer function are then compared to the identified SISO linear to determine the unknown parameters. Although other researchers have used MIMO identification techniques ${ }^{1,6}$ to find stability derivatives, SISO identification is better suited for our purpose as we are interested in identifying actual physical parameters.

Similar to the approach presented in ref. 33, the system identification is performed in the discretetime domain using input-output data taken by flying the helicopter on the stands. The coefficients of a discrete-time transfer function of the same order as the continuous-time transfer function are identified using the direct least squares method. The transfer function is then converted into state space form, where the matrix logarithm method with zero-order hold is used to directly convert into the corresponding continuous-time transfer function. By keeping all the parameters numerical, we can avoid dealing with the nonlinear coefficients which arise when the continuous-time transfer function is converted into the equivalent discrete-time transfer function analytically.

A Polhemus sensor ${ }^{34}$ is used to measure the position and orientation of the helicopter: full six degree-of-freedom information $\left(x_{I}, y_{I}, z_{I}, \phi, \theta, \psi\right)$ is

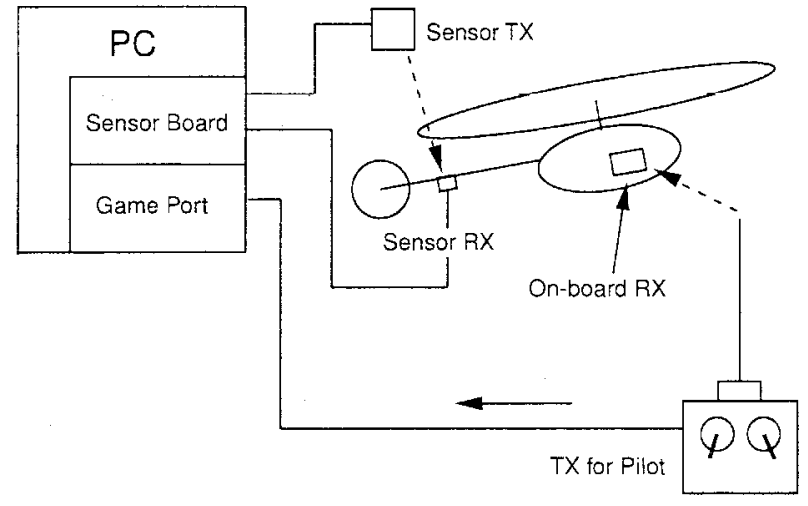

Figure 10. A sketch of the experimental setup for the system identification of the helicopter's physical parameters. The helicopter is controlled through the radio transmitter by a human pilot. The computer is used to record the input data from the transmitter and the output data from the sensor simultaneously.

output data are taken at $50 \mathrm{~Hz}$ for a total of approxiavailable at $50 \mathrm{~Hz}$. As shown in Figure 10, the sensor consists of a board connected to the PC's ISA slot, a transmitter, and a receiver. The transmitter is fixed to the earth and sends out a magnetic field via three orthogonal inductors. The receiver, fixed to the helicopter, senses the strength and the orientation of the magnetic field and sends this information back to the PC. The sensor has a static accuracy of $0.5 \mathrm{in}$. rms for the $x_{I}, y_{I}$, and $z_{I}$ position and 2 degrees rms for the $\phi, \theta$, and $\psi$ orientation. To obtain a reliable SISO ID result, we found that it is important to carefully design a stand which allows the helicopter to move in one direction for each ID session. Four different stands were used separately for each degree of freedom; they are shown in Figure 11. Each stand was constructed to be sturdy yet light-weight to minimize the influence of the stand dynamics. The input and output data are taken at $50 \mathrm{~Hz}$ for a total of

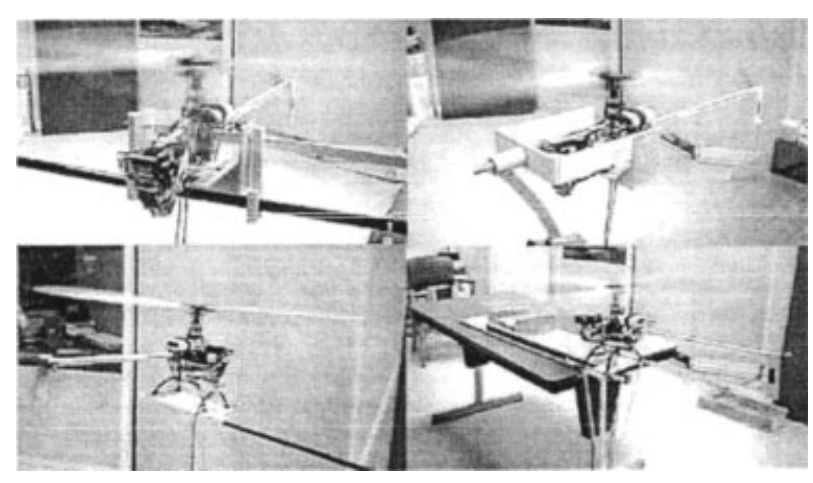

Figure 11. Separate identification was done for each degree of freedom. From top left, clockwise, they are pitch, roll, yaw, and heave identification. 
approximately $40 \mathrm{~s}$ duration for each degree of freedom. Due to the difficulty of maintaining an exact trim at the beginning of data collection, the helicopter is simply disturbed around an upright trim position. However, applying the system identification algorithm at this point could lead to an incorrect result due to the possibility that the collected data is disturbed around a nonzero trim. A linear identification model should be based on an assumption that the collected data is disturbed exactly from trim. To solve this problem, we first define the collected input and output values as $X^{c}$, trim values as $\bar{X}$, and disturbed data as $\hat{X}$. We can then write

$$
X^{c}=\bar{X}+\hat{X} \text {. }
$$

Although finding the exact value of $\bar{X}$ can be difficult, since the helicopter is disturbed around a trim with upright position, the average of $X^{c}$ should be nearly equal to $\hat{X}:{ }^{35}$

$$
\bar{X} \approx \frac{1}{N} \sum_{k=1}^{N} X^{c}(k) .
$$

The above should be even more true when the data is collected for a sufficiently long time. We can therefore obtain $\hat{X}$ from (27) and (28) for our identification:

$$
\hat{X}=X^{c}-\frac{1}{N} \sum_{k=1}^{N} X^{c}(k) .
$$

Once the input-output data have been taken and stored for the isolated single DOF motion, the data is preprocessed using Eq. (29). The system identification algorithm is then run to estimate the discretetime transfer function coefficients. The initial condition of the system is also estimated using a leastsquares method. The input data and the estimated transfer function are used to simulate the output data, and the simulated and actual output are compared. Ten independent identification sessions were run with similar results; the numerical results in Sections $4.1-4.3$ are the results of one of these sessions.

\subsection{Pitch and Roll Identification}

The main purpose of the pitch and roll identification is to find the inertia terms $I_{x x}$ and $I_{y y}$ used in Eq. (2),although the coefficients $\alpha_{1}$ and $\alpha_{2}$ must also be determined. The derivation will be done here for the roll equation; the pitch equations are similar. First, we substitute the approximation $\beta \approx \beta_{\max } \cos \left(\xi-\xi_{\max }\right)$ from Eq. (26) into the roll-restricted equation for the flybar flapping angle $\beta$ given by (18), resulting in the following expression:

$$
\begin{gathered}
-\cos \xi \ddot{\phi}+2 \Omega \sin \xi \dot{\phi}-\sin ^{2} \xi \beta_{\max } \cos \left(\xi-\xi_{\max }\right) \dot{\phi}^{2} \\
=P_{1} P_{2} \alpha_{1} \delta_{\phi} \sin \xi+\alpha_{2} P_{1} \beta_{\max } \sin \left(\xi-\xi_{\max }\right) \\
+\frac{\alpha_{2} P_{1}}{\Omega} \dot{\phi} \cos \xi
\end{gathered}
$$

where, to simplify the notation somewhat, the terms $P_{1}$ and $P_{2}$ are defined as follows:

$$
\begin{gathered}
P_{1}=\frac{n \Omega^{2}}{8} \frac{\rho a c_{2}\left(B^{4} R_{2}^{4}-R_{1}^{4}\right)}{I_{f}} \\
P_{2}=\frac{L_{7} L_{8}}{L_{5} L_{6} L_{9}} .
\end{gathered}
$$

Note that $P_{1}$ and $P_{2}$ can be determined by direct measurements of helicopter parameters. The lift curve slope $a$ is taken to be 6 (ref. 23) and the flybar inertia $I_{f}$ is computed using basic physics and the mass of the flybar.

Now, we collect terms in sine and cosine from the roll equation, resulting in

$$
\begin{gathered}
C_{1} \cos \left(\xi-\xi_{\max }\right)+C_{2} \sin \left(\xi-\xi_{\max }\right) \\
=C_{3} \cos \xi+C_{4} \sin \xi
\end{gathered}
$$

where, again, new notation has been introduced to simplify the expression:

$$
\begin{gathered}
C_{1}=-\beta_{\max } \sin ^{2} \xi \dot{\phi}^{2}, \\
C_{2}=-\alpha_{2} P_{1} \beta_{\max } \\
C_{3}=\ddot{\phi}+\frac{\alpha_{2} P_{1}}{\Omega} \dot{\phi}, \\
C_{4}=-2 \Omega \dot{\phi}+\alpha_{1} P_{1} P_{2} \delta_{\phi} .
\end{gathered}
$$

Applying the trigonometric identity,

$$
\begin{gathered}
A_{1} \cos \xi+A_{2} \sin \xi=\sqrt{A_{1}^{2}+A_{2}^{2}} \sin (\xi+\gamma), \\
\gamma=\arctan \frac{A_{1}}{A_{2}},
\end{gathered}
$$


Eq. (30) can be rewritten as

$$
\begin{gathered}
\sqrt{C_{1}^{2}+C_{2}^{2}} \sin \left(\xi-\xi_{\max }+\arctan \frac{C_{1}}{C_{2}}\right) \\
=\sqrt{C_{3}^{2}+C_{4}^{2}} \sin \left(\xi+\arctan \frac{C_{3}}{C_{4}}\right),
\end{gathered}
$$

which implies that the magnitude and phase terms on both sides of the equation should match:

$$
\begin{gathered}
C_{1}^{2}+C_{2}^{2}=C_{3}^{2}+C_{4}^{2}, \\
-\xi_{\max }+\arctan \frac{C_{1}}{C_{2}}=\arctan \frac{C_{3}}{C_{4}} .
\end{gathered}
$$

Our system ID experiment suggests terms involving $\ddot{\phi}^{2}, \ddot{\phi} \dot{\phi}, \dot{\theta}^{4}$ and their coefficients are small in general. Based on this fact, solving for $\beta_{\max }^{2}$ yields the following expression:

$$
\beta_{\max }^{2} \approx\left(\frac{\alpha_{1} P_{2}}{\alpha_{2}} \delta_{\phi}\right)^{2}-4 \frac{\alpha_{1} P_{2} \Omega}{\alpha_{2}^{2} P_{1}} \delta_{\phi} \dot{\phi}+\left(\frac{1}{\Omega^{2}}+\frac{4 \Omega^{2}}{\alpha_{2}^{2} P_{1}^{2}}\right) \dot{\phi}^{2} .
$$

Neglecting $1 / \Omega^{2}$, which is also small, we get a perfect square, and use the approximation

$$
\beta_{\max } \approx \frac{\alpha_{1} P_{2}}{\alpha_{2}} \delta_{\phi}-\frac{2 \Omega}{\alpha_{2} P_{1}} \dot{\phi}
$$

Next, we need to solve for $\xi_{\max }$. Inspecting the magnitudes and signs of $C_{1}-C_{4}$, we can determine that, for roll motion,

$$
\begin{aligned}
& \arctan \frac{C_{1}}{C_{2}} \approx \pi, \\
& \arctan \frac{C_{3}}{C_{4}} \approx 0,
\end{aligned}
$$

and, therefore,

$$
\xi_{\max } \approx \pi
$$

We now substitute $\beta_{\max }$ and $\xi_{\max }$ into the expression for $M_{\phi}$ from (21),

$$
\begin{aligned}
M_{\phi}= & \left(\frac{\alpha_{3} P_{3} L_{3} L_{8}}{L_{9}}+\frac{\alpha_{1} \alpha_{3}}{\alpha_{2}} P_{2} P_{3} L_{2} L_{4}\right) \delta_{\phi} \\
& -\left(\frac{2 \alpha_{3} P_{3} L_{2} L_{4} \Omega}{\alpha_{2} P_{1}}+\frac{\alpha_{4} P_{3} L_{1}\left(L_{2}+L_{3}\right)}{\Omega}\right) \dot{\phi}
\end{aligned}
$$

where

$$
P_{3}=\frac{n \rho a c \Omega^{2} R^{4} B^{4}}{16 L_{1}\left(L_{2}+L_{3}\right)}
$$

can be measured directly.

We now have an analytic coefficient for a simplified linear transfer function describing SISO roll dynamics:

$$
\frac{\Phi(s)}{\delta_{\phi}(s)}=\frac{\left(\alpha_{3} P_{3} L_{3} L_{8} / L_{9}+\left(\alpha_{1} \alpha_{3} / \alpha_{2}\right) P_{2} P_{3} L_{2} L_{4}\right) 1 / I_{x x}}{s\left(s+\left(2 \alpha_{3} P_{3} L_{2} L_{4} \Omega / \alpha_{2} P_{1}+\alpha_{4} P_{3} L_{1}\left(L_{2}+L_{3}\right) / \Omega\right) 1 / I_{x x}\right)} .
$$

We now have a transfer function with parameters to be identified by comparing with a numerical identification. However, the expression contains not only $I_{x x}$, but also $\alpha$. We therefore need to determine appropriate $\alpha$ before finding $I_{x x}$. This task is not straightforward because $\alpha, I_{x x}, I_{y y}$ and the 6-DOF simulation all depend on each other. One can nevertheless find a suitable set of parameters to obtain a reasonable solution.

An ID was carried out to obtain a discrete transfer function $H_{\phi}(z)$ and $H_{\theta}(z)$, with two poles and two zeros. Before ID, the roll output data was filtered using a first-order low-pass Butterworth filter with the cutoff frequency at $1.5 \mathrm{~Hz}$ :

$$
H_{\phi}(z)=\frac{0.559 z^{2}}{z^{2}-1.81 z+0.816} .
$$

This transfer function is then converted into an equivalent continuous transfer function $H_{\phi}(s)$, using 

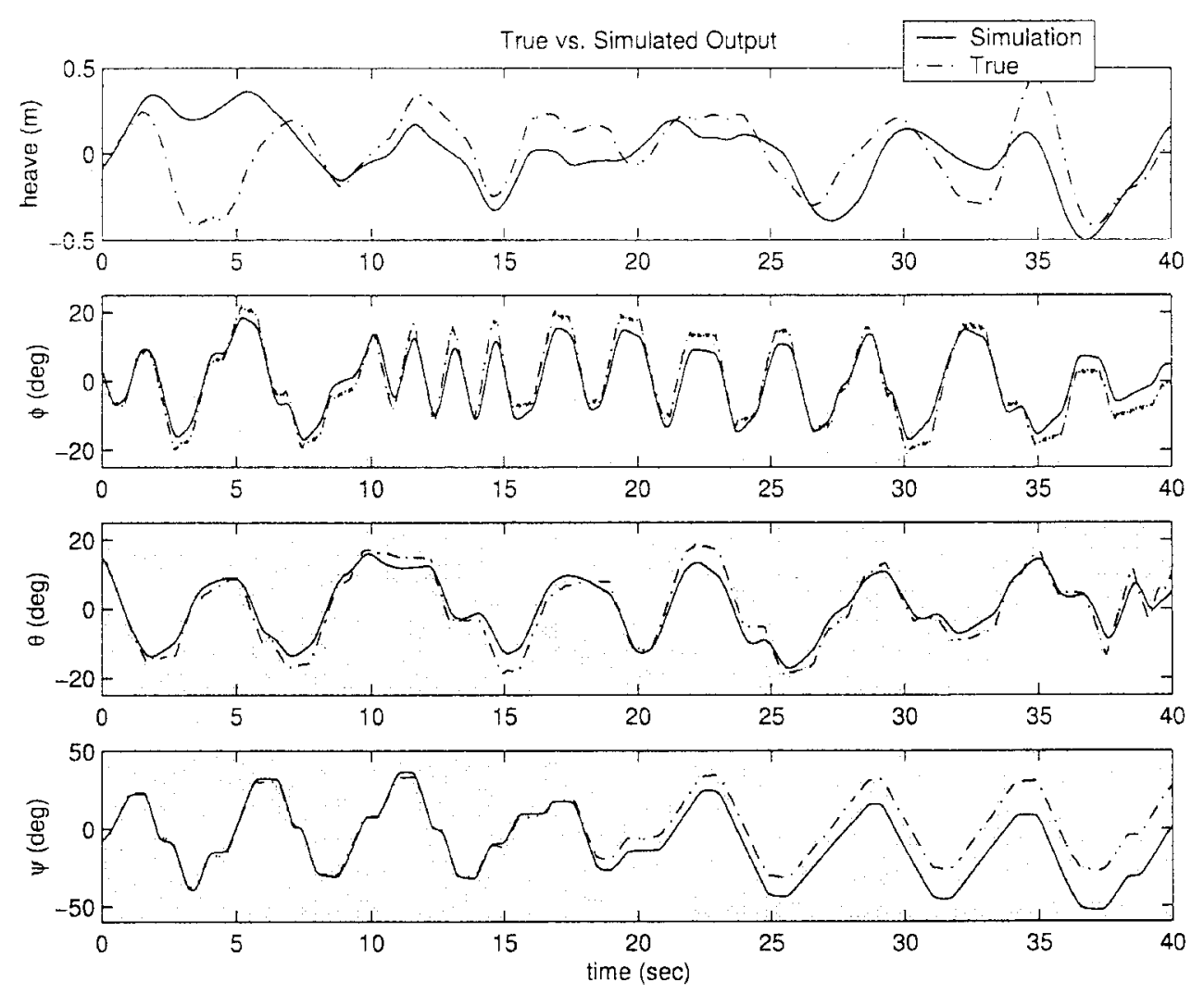

Figure 12. The comparison between the simulated outputs based on the identified discrete time transfer functions and the actual outputs from the experiment, given the identical input command history separately. The initial values for the simulation was also determined with least squares method using the first 50 points from the experiment.

a matrix logarithm method with zero-order hold. Ignoring small coefficients for the numerator and assuming the pole at $s=-0.503$ to be at the origin,

$$
\frac{\Phi(s)}{\delta_{\phi}(s)}=\frac{0.559 s^{2}+45.8 s+1540}{s^{2}+10.2 s+4.85} \approx \frac{1540}{s(s+9.65)}
$$

We apply the same technique for the pitch motion; the pitch output data was filtered using a firstorder low-pass Butterworth filter with the cutoff frequency at $1.0 \mathrm{~Hz}$ :

$$
\begin{gathered}
H_{\theta}(z)=\frac{0.234 z^{2}}{z^{2}-1.92 z+0.922} \\
\frac{\Theta(s)}{\delta_{\theta}(s)}=\frac{0.234 s^{2}+18.2 s+610}{s^{2}+4.04 s+0.946} \approx \frac{610}{s(s+3.80)} .
\end{gathered}
$$

The inertia terms can now be calculated by applying the identified numerical values into (32). However, we now have two equations for one unknown and they do not necessarily yield a single solution. We therefore use averaged values for each motion, to determine

$$
\begin{aligned}
& I_{x x}=0.137 \mathrm{~kg} \cdot \mathrm{m}^{2}, \\
& I_{y y}=0.221 \mathrm{~kg} \cdot \mathrm{m}^{2} .
\end{aligned}
$$

Meanwhile, a reasonable match is achieved between the true data and $H(z)$, as shown in Figure 12. For the roll motion ID, the mean-squared error between the actual output $\phi_{a}$ and the simulated output $\phi_{s}$ from the estimated transfer function is computed as follows:

$$
\text { rms error }=\sqrt{\frac{1}{N} \sum_{i=1}^{N}\left(\phi_{a}(i)-\phi_{s}(i)\right)^{2}}
$$



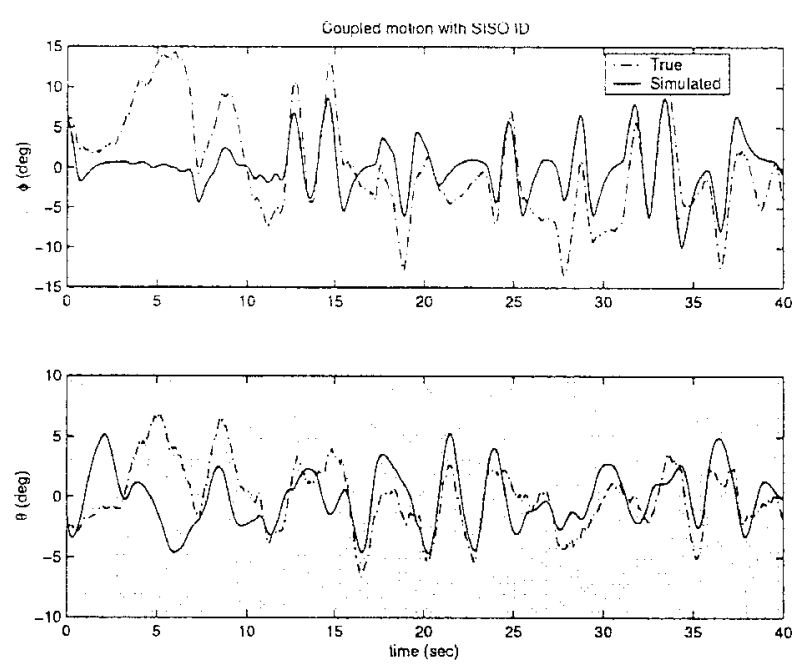

Figure 13. The system identification result is applied to the pitch and roll motion simultaneously. The figure shows some coupled responses through the discrepancy between the true and the simulated result.

Typical values for this error are on the order of a few degrees, which is of the same order as the accuracy of the sensor.

To determine the validity of the single-axis identification, an experiment was performed in which both pitch and roll motions were commanded by the pilot. The input-output data were collected, and the input data were used with the single-input transfer functions to simulate both the pitch and roll outputs. The results from this experiment are given in Figure 13. There is some coupling between the pitch and roll motions, but it does not overwhelm the dominant single-input effects. A sampling of the mean-squared errors is given in Table I.

Table I. A sampling of the mean-squared errors between the actual and simulated outputs using the identified transfer functions $H_{\phi}(z), H_{\theta}(z), H_{z}(z)$, and $H_{\psi}(z)$. Although the errors are slightly larger in the coupled experiment, they are still small enough to give some degree of confidence in the identified transfer function.

\begin{tabular}{lc}
\hline Experiment & RMS error \\
\hline pitch SISO & $2.9^{\circ}$ \\
roll SISO & $3.3^{\circ}$ \\
yaw SISO & $13^{\circ}$ \\
heave SISO & $0.22 \mathrm{~m}$ \\
pitch coupled & $2.9^{\circ}$ \\
roll coupled & $5.4^{\circ}$ \\
\hline
\end{tabular}

\subsection{Yaw Identification}

By comparing $H_{\psi}(z)$ with a second-order yaw dynamic model from Eq. (2), we can obtain parameters such as $I_{z z}$ and $K_{g}$ which are uniquely related to the yaw dynamics. We first rewrite the yaw dynamic equation for hover by considering the yaw moment input $M_{\psi}$ and yaw angle output $\psi$ only:

$$
I_{z z} \dot{\omega}_{B 3}=M_{\psi}-\tau_{m}=M_{\delta_{\psi}}-K_{g} \omega_{B 3}-\tau_{m},
$$

where we have defined $M_{\delta_{\psi}}$ as $T_{T} L_{T}$. For the reaction moment $\tau_{m}$,

$$
\tau_{m}=-K_{m} \cdot \delta_{o}
$$

the proportional constant $K_{m}$ can be found by directly measuring $\tau_{m}$ using a small spring scale with respect to several different values of $\delta_{o}$, and results in

$$
K_{m}=0.178 \mathrm{~N} \cdot \mathrm{m} / \mathrm{deg} .
$$

We then linearize about hover, using the reference value $\delta_{\psi}=0$ when there is no yaw moment, to get

$$
\begin{gathered}
I_{z z} \ddot{\psi}=M_{\delta_{\psi}}-K_{g} \dot{\psi}, \\
\frac{\Psi(s)}{M_{\delta_{\psi}}(s)}=\frac{1 / I_{z z}}{s\left(s+K_{g} / I_{z z}\right)} .
\end{gathered}
$$

Before ID, the output data was filtered using a fifth-order low-pass Butterworth filter with the cutoff frequency at $5 \mathrm{~Hz}$. After using the identification procedure similar to the roll and pitch case, and ignoring the near-infinite zero at $s=-104$ and assuming the pole at $s=-0.0245$ to be at the origin,

$$
\frac{\Psi(s)}{M_{\delta_{\psi}}(s)}=\frac{0.289 s+30.1}{s^{2}+12.3 s+0.301} \approx \frac{30.1}{s(s+12.3)} .
$$

We can then find the parameters,

$$
\begin{gathered}
I_{z z}=0.0332 \mathrm{~kg} \cdot \mathrm{m}^{2}, \\
K_{g}=0.408 \mathrm{~kg} \cdot \mathrm{m} .
\end{gathered}
$$


Table II. List of parameters identified using the system identification method for ten independent runs. Average and standard deviation values are reported.

\begin{tabular}{lc}
\hline Parameter & Average values with standard deviation \\
\hline$I_{x x}$, Roll Inertia & $0.137 \pm 0.0228\left[\mathrm{~kg} \cdot \mathrm{m}^{2}\right]$ \\
$I_{y y}$, Pitch Inertia & $0.221 \pm 0.0478\left[\mathrm{~kg} \cdot \mathrm{m}^{2}\right]$ \\
$I_{z z}$, Yaw Inertia & $0.0323 \pm 0.00234\left[\mathrm{~kg} \cdot \mathrm{m}^{2}\right]$ \\
$K_{g}$, Gyro Gain & $0.400 \pm 0.0104[\mathrm{~kg} \cdot \mathrm{m}]$ \\
$K_{1}$ & $0.886 \pm 0.0954\left[\mathrm{~N} \cdot \mathrm{s} \cdot \mathrm{kg}^{-1} \cdot \mathrm{m}^{-1}\right]$ \\
$K_{2}$ & $1.12 \pm 0.250\left[\mathrm{~N} \cdot \mathrm{kg}^{-1} \cdot \mathrm{m}^{-1}\right]$ \\
$K_{3} K_{4}$ & $442000 \pm 73200\left[\mathrm{~N}^{2} \cdot \mathrm{kg}^{-2} \cdot \mathrm{deg}^{-2}\right]$ \\
$K_{5}$ & $27.7 \pm 1.20\left[\mathrm{~N} \cdot \mathrm{s} \cdot \mathrm{kg}^{-1} \cdot \mathrm{deg}^{-1}\right]$ \\
$K_{6}$ & $5760 \pm 302\left[\mathrm{~N} \cdot \mathrm{kg}^{-1} \cdot \mathrm{deg}^{-1}\right]$ \\
\hline
\end{tabular}

Based on the Equation (25), we first need to find the relationship between $\delta_{\psi}$ and $M_{\delta_{\psi}}$ to be able to use the yaw dynamics model given above. We ignore the effect of $\lambda_{T}$, then directly measure the yaw moment per several different values of $\delta_{\psi}$ using a simple spring scale. That is,

$$
M_{\delta_{\psi}} \approx 0.0474[\mathrm{~N} \cdot \mathrm{m} / \mathrm{deg}] \delta_{\psi} .
$$

\subsection{Heave Identification}

We can also simplify the heave motion dynamic model from Eqs. (2) and (20) for the hovering condition. Selecting a nominal value of $\theta_{0}$ at hover gives the equation

$$
\ddot{z}+K_{1} \dot{z}+K_{2} z=K_{3} \theta_{0}
$$

where,

$$
\begin{aligned}
& K_{1}=\text { coefficient } \text { for simplified } \lambda, \\
& K_{2}=\text { coefficient for ground effect, }
\end{aligned}
$$

$$
K_{3}=\frac{n a c \rho \pi R^{3} \Omega^{2} B^{3}}{6 m}
$$

\begin{tabular}{|c|c|c|c|}
\hline Parameter & Value & Parameter & Value \\
\hline$a$ & 6 & $L_{T}$ & $0.635 \mathrm{~m}$ \\
\hline$\alpha$ & {$\left[\begin{array}{llll}0 & 3 & 0.3 & 1\end{array}\right]$} & $L_{1}$ & $0.0190 \mathrm{~m}$ \\
\hline$B$ & 0.97 & $L_{2}$ & $0.0119 \mathrm{~m}$ \\
\hline$c$ & $0.044 \mathrm{~m}$ & $L_{3}$ & $0.0098 \mathrm{~m}$ \\
\hline$c_{2}$ & $0.038 \mathrm{~m}$ & $L_{4}$ & $0.0250 \mathrm{~m}$ \\
\hline$g$ & $9.8 \mathrm{~m} / \mathrm{s}^{2}$ & $L_{5}$ & $0.0177 \mathrm{~m}$ \\
\hline$I_{f}$ & $0.00128 \mathrm{~kg} \cdot \mathrm{m}^{2}$ & $L_{6}$ & $0.0110 \mathrm{~m}$ \\
\hline$I_{x x}$ & $0.137 \mathrm{~kg} \cdot \mathrm{m}^{2}$ & $L_{7}$ & $0.0234 \mathrm{~m}$ \\
\hline$I_{y y}$ & $0.221 \mathrm{~kg} \cdot \mathrm{m}^{2}$ & $L_{8}$ & $0.0175 \mathrm{~m}$ \\
\hline$I_{z z}$ & $0.0323 \mathrm{~kg} \cdot \mathrm{m}^{2}$ & $L_{9}$ & $0.0266 \mathrm{~m}$ \\
\hline$K_{g}$ & $0.400 \mathrm{~kg} \cdot \mathrm{m}$ & $m$ & $1.36 \mathrm{~kg}$ \\
\hline$K_{m}^{\circ}$ & $0.178 \mathrm{~N} \cdot \mathrm{m} / \mathrm{deg}$ & $R$ & $0.508 \mathrm{~m}$ \\
\hline$K_{1}$ & $0.886 \mathrm{~N} \cdot \mathrm{s} \cdot \mathrm{kg}^{-1} \cdot \mathrm{m}^{-1}$ & $R_{1}$ & 0.15 \\
\hline$K_{2}$ & $1.12 \mathrm{~N} \cdot \mathrm{kg}^{-1} \cdot \mathrm{m}^{-1}$ & $R_{2}$ & 0.21 \\
\hline$K_{3} K_{4}$ & $442000 \mathrm{~N}^{2} \cdot \mathrm{kg}^{-2} \cdot \mathrm{deg}^{-2}$ & $\rho$ & $1.2 \mathrm{~kg} / \mathrm{m}^{3}$ \\
\hline$K_{5}$ & $27.7 \mathrm{~N} \cdot \mathrm{s} \cdot \mathrm{kg}^{-1} \cdot \mathrm{deg}^{-1}$ & $\Omega$ & 1190 RPM \\
\hline$K_{6}$ & $5760 \mathrm{~N} \cdot \mathrm{kg}^{-1} \cdot \mathrm{deg}^{-1}$ & & \\
\hline
\end{tabular}

Since our identification was done near ground environment, the approximated ground effect, $K_{2} z$, was not negligible. Further investigations indicated

Table III. The following values are used for the simulation. 
that there was significant mechanical compliance in the collective pitch control system, and Eq. (19) was added to the system model:

$$
\frac{\Theta_{o}(s)}{\delta_{o}(s)}=\frac{K_{4}}{s^{2}+K_{5} s+K_{6}} .
$$

Combining the equations (19) and (33), we arrived at a fourth-order continuous-time transfer function;

$$
\frac{Z(s)}{\delta_{0}(s)}=\frac{K_{3} K_{4}}{\left(s^{2}+K_{1} s+K_{2}\right)\left(s^{2}+K_{5} s+K_{6}\right)} .
$$

Similar to the yaw case, we identified the discrete transfer function $H_{z}(z)$ with four poles and four zeros, then converted this into a continuous transfer function. Before ID, the input data was filtered using a third-order low-pass Butterworth filter with the cutoff frequency at $5 \mathrm{~Hz}$. The output data was filtered with first order at $10 \mathrm{~Hz}$. Ignoring the small coefficients in the numerator,

$$
\begin{aligned}
\frac{Z(s)}{\delta_{0}(s)} & =\frac{-0.887 s^{3}+129 s^{2}-10131 s+326000}{s^{4}+29.6 s^{3}+5030 s^{2}+4740 s+5070} \\
& \approx \frac{326000}{\left(s^{2}+0.942 s+1.01\right)\left(s^{2}+28.7 s+5004\right)} .
\end{aligned}
$$

\subsection{Identification Summary}

Note that one could simply use the identified transfer functions from the roll, pitch, yaw, and heave identification directly without going through the simplification presented in the last sections. However, those ignored or neglected poles and zeros basically represent unmodelled dynamics not included in Eq. (2). Therefore, those terms do not guarantee the usefulness for all operating conditions.

Standard deviations for identified parameters were determined by repeating the identification session ten times. Input and output data were collected for $10 \mathrm{~min}$, then divided into ten sections for ten different ID sessions. Table II summarizes all the parameters identified.

\section{COMPARISON BETWEEN THE NONLINEAR AND LINEAR HELICOPTER MODEL}

As mentioned in Section 2, there exist a number of linear helicopter models that can adequately represent helicopter dynamics near hover condition. In this section, we will compare our nonlinear model with the linear model presented in ref. 1, and demonstrate how the nonlinear model is able to represent the dynamics of the helicopter at angular positions well beyond the nominal hover region. The values used in the simulation are given in Table III. Note the model in ref. 1 is based on a Yamaha R-50 industrial helicopter with a 10 -foot rotor span, which is much larger than our Ikarus helicopter with a 3-foot rotor span. However, we can still see the fundamental difference between the two models in the following.

Both starting from a hover condition, we apply a pitch input $\delta_{\theta}$ for $1 \mathrm{~s}$ to increase the pitch angle $\theta$ to about $45^{\circ}$, then a yaw input $\delta_{\phi}$ is applied for $1 \mathrm{~s}$ to rotate the helicopter $90^{\circ}$ clockwise. All other inputs remain constant. As shown in Figure 14, after those two inputs are applied, the roll angle $\phi$ is now near $45^{\circ}$ and the pitch angle is now near zero. Therefore, due to the nonlinearity involved with the rigid body dynamics, there is an "angle-reversal" between $\theta$ and $\phi$. When similar inputs are applied to the linear model by ref. 1, such coupling effect is almost absent. As shown in Figure 15, the roll and pitch angles change only little with respect to the changing yaw angle. In fact, this result is common to all other linear helicopter models. This comparison implies the necessity of a nonlinear model when a helicopter is performing aggressive maneuver, which often requires large control inputs and large deviations from the hover condition.

\section{CONCLUSIONS}

In this paper, we have derived a complete mathematical model for a model-scale helicopter robot, experimentally identified the key parameters of the model, and verified the model through simulation. The main contribution of the work is the model of interaction between the flybar and the main rotor blade. A flybar is typically used on model-scale helicopters to augment the stability and aid in actuation. Understanding the dynamics of the rotor hub is an essential part of modeling a helicopter robot. The nonlinear flybar modeling presented should provide an insight into the delicate mechanism which should provide a way to improve the existing mechanism for better stability and control characteristics for different helicopter 

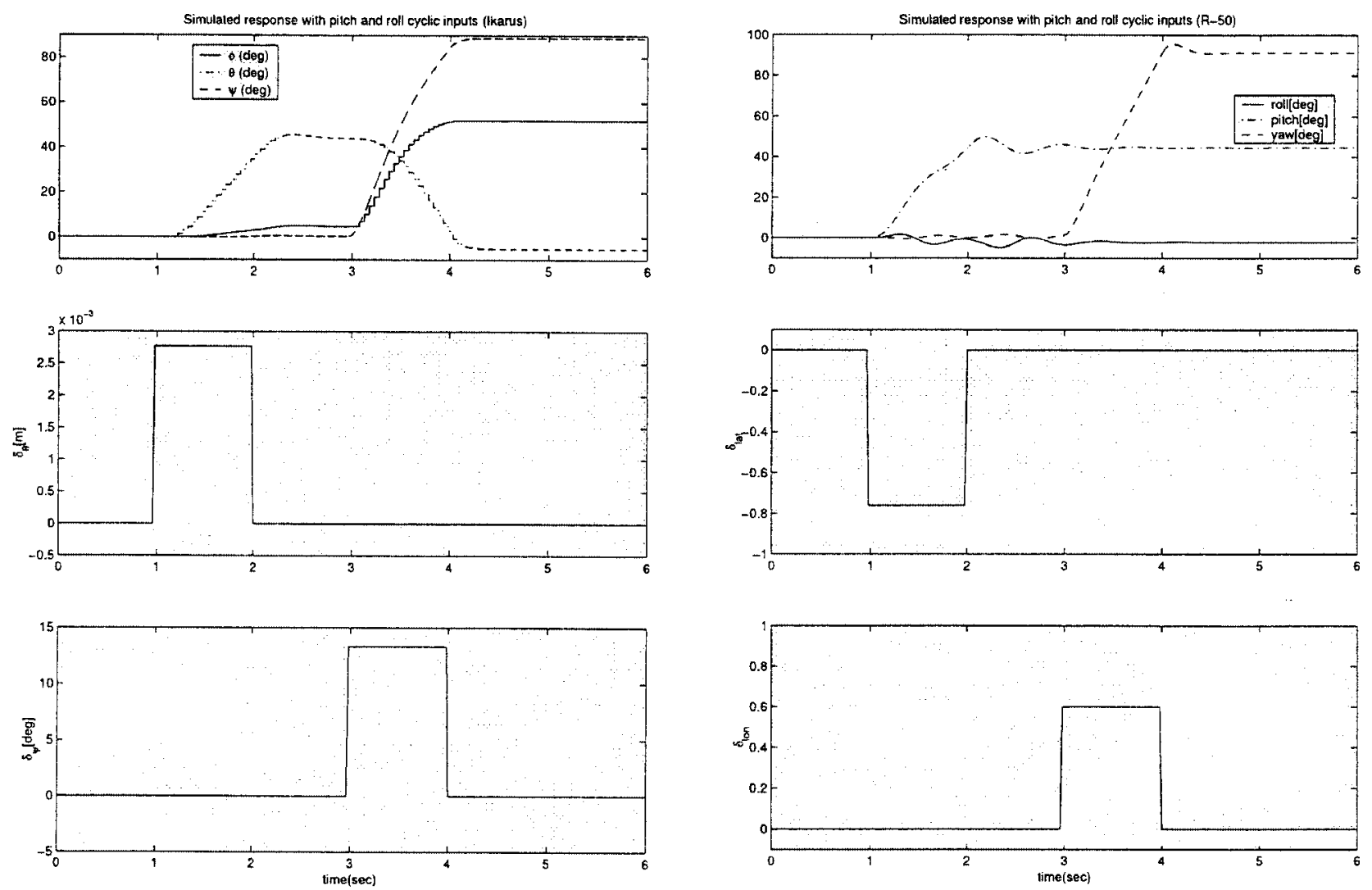

Figure 14. The simulated angular response of the Ikarus helicopter with respect to our nonlinear model when pitch and yaw input are applied sequentially. Even when there is no roll input, the helicopter is rolled $45^{\circ}$ after the inputs are applied.

robots. Furthermore, when the robot is maneuvered beyond near hover attitude with large cyclic control input, significant coupling between each Euler angle demands a nonlinear modeling to better predict the motion of the helicopter.

The result of this work will be of interest to researchers and developers working in the increasingly important area of small, autonomous aerial vehicles.

\section{NOMENCLATURE}

$a$ main rotor lift slope

$a_{T}$ tail rotor lift slope

$B$ tip loss factor

$c, c_{2}, c_{T}$ main/flybar/tail blade chord length

$D_{F_{x y, z}}$ fuselage profile drag forces along body coordinates

$d L, d L_{m}$ differential lift elements for flybar and main rotor blade

$d r$ differential length elements for flybar and main rotor blade

$f_{B}$ external force applied along body coordinates

Figure 15. The simulated angular response of the Yamaha R-50 helicopter with respect to the linear model of ref. 1 when pitch and yaw inputs are applied sequentially. There are only slight couplings between each angle as per numerical identification. Required large coupling effects between the roll and pitch angles are absent.

$g$ gravitational acceleration

$h_{r}$ distance between rotor disk and CG, parallel to rotor axis

$\hat{I}$ rotational inertia matrix of flybar

$I_{3 \times 3} \quad 3 \times 3$ identity matrix

$I_{f}$ flybar moment of inertia in flapping

$I_{x x, y y, z z, x z}$ fuselage rotational moments of inertia

I rotational inertia matrix of helicopter

$K_{g}$ gyro gain for tail rotor

$K_{m}$ motor reaction torque gain

$K_{\Omega}$ proportional constant relating $\Omega$ to $\Omega_{T}$

$K_{1}, \ldots, K_{6}$ proportional constants used for identification

$\ell_{r}$ distance between rotor axis and CG, perpendicular to rotor axis

$L_{T}$ distance between tail rotor axis and CG

$L_{1}, \ldots, L_{9}$ linkage lengths in rotor hub assembly

$m$ helicopter total mass

$m_{F}$ Flybar mass per length

$M_{\phi, \theta, \psi}$ net moments on helicopter

$M_{\delta_{\psi}}$ yaw moment due to pilot input

$n$ number of main/flybar/tail blades

$r$ position along the main rotor blade 


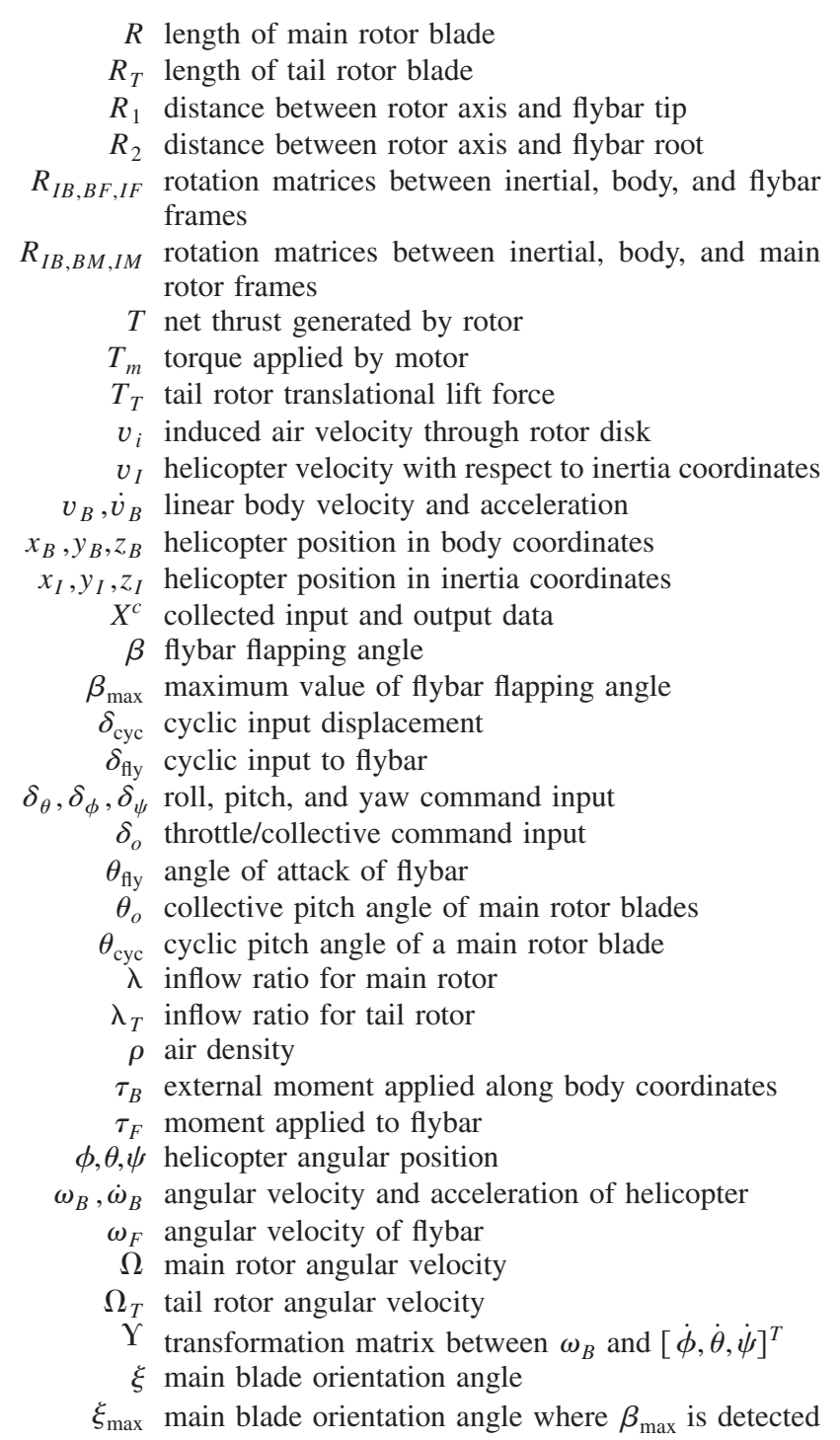

\section{REFERENCES}

1. B. Mettler, M. Tischler, and T. Kanade, System identification modeling of a model-scale helicopter, Tech. Rep. CMU-RI-TR-00-03, Robotics Institute, Carnegie Mellon University, Pittsburgh, PA, January 2000.

2. E. Lee, H. Shim, H. Park, and K. Lee, Design of hovering attitude controller for a model helicopter, in Proc of Society of Instrument and Control Engineers, August 1993, pp. 1385-1389.

3. T. Pallett, Real-time helicoper flight control, Master's thesis, School of Electrical Engineering, Purdue University, August 1991.

4. K. Furuta, Y. Ohyama, and O. Yamano, Dynamics of rc helicopter and control, Mathematics and Computers in Simulation XXVI, 1984, 148-159.

5. M. Weilenmann and H. Geering, Test bench for rotorcraft hover control, AIAA J Guidance Control Dynamics 17:(4) (1994), 729-736.
6. J. Morris, M. Nieuwstadt, and P. Bendotti, Identification and control of a model helicopter in hover, in Proc of the American Control Conf, 1994, Vol. 2, pp. 12381242.

7. X. Zhu and M. Nieuwstadt, The Caltech helicopter control experiment, CDS Technical Report 96-009, 1996.

8. D. Teare, P. FitzSimons, and B. Tongue, Time domain parameter identification techniques applied to the $\mathrm{UH}$ 60A black hawk helicopter, Vertica 13:(3) (1989), 393401.

9. F. Kim, R. Celi, and M. Tischler, High-order state space simulation models of helicopter flight mechanics, J Am Helicopter Soc 38:(4) (1993), 16-27.

10. F. Kim, Formulation and validation of high-order mathematical models of helicopter flight dynamics, PhD thesis, University of Maryland, 1991.

11. R. Chen, A simplified rotor system mathematical model for piloted flight dynamics simulation, NASA technical memorandum 78575, NASA Ames Research Center.

12. P. Sheridan, C. Robinson, J. Shaw, and F. White, Mathematical modeling for helicopter simulation of low speed, low altitude, and steeply descending flight, NASA contractor report 166385, NASA Ames Research Center.

13. P. Talbot, B. Tinling, W. Decker, and R. Chen, A mathematical model of a single main rotor helicopter for piloted simulation, NASA technical memorandum 84281, NASA Ames Research Center.

14. A. Azuma, Dynamic analysis of the rigid rotor system, J Aircraft 4:(3) (1967), 203-209.

15. W. Warmbrodt and P. Friedmann, Formulation of coupled rotor/fuselage equations of motion, Vertica 3:(3-4) (1979), 245-271.

16. D. Menaker and A. Rosen, A model for helicopter performance calculation, Vertica 12:(1-2) (1988), 155-178.

17. M. Rohlfs, Identification of non-linear derivative models from BO-105 flight test data, Aeronaut J 102:(1011) (1998), 1-8.

18. M. Tischler, Advancements in frequency-domain methods for rotorcraft system identification, Vertica 13:(3) (1989), 327-342.

19. J. Fay, The helicopter: history, piloting, and how it flies, 3rd rev. ed., Newton Abbot, North Pomfret, VT., David \& Charles, 1976.

20. E. Johnson and P. DeBitetto, Modeling and simulation for small autonomous helicopter development, in Proc of the AIAA Modeling and Simulation Technologies Conf, 1997, 41-51.

21. A. Bramwell, Helicopter dynamics, Halsted, New York, 1976.

22. R. Murray, Z. Li, and S. Sastry, A mathematical introduction to robotic manipulation, CRC, Boca Raton, FL, 1994.

23. W. Johnson, Helicopter theory, Dover, New York, 1980.

24. G. Padfield, Helicopter flight dynamics, Blackwell Science, Oxford, 1996.

25. Hobby Lobby Intl., 5614 Franklin Pike Cr, Brentwood, TN 37027, Ikarus ECO electric helicopter.

26. Aveox Inc., 31324 Via Colinas, Suite 103, Westlake Village, CA 91362, 1406/2Y Brushless Motor.

27. T. Koo and S. Sastry, Output tracking control design 
of a helicopter model based on approximate linearization, in Proc of the IEEE Conf on Decision and Control, 1998.

28. Y. Okuno, K. Kawachi, A. Azuma, and S. Saito, Analytical prediction of height-velocity diagram of a helicopter using optimal control theory, AIAA J Guidance Control Dynamics 14:(2) (1991), 453-459.

29. R. Shevell, Fundamentals of flight, Prentice-Hall, Englewood Cliffs, NJ, 1989.

30. D. Schlüter, Schlüter's radio controlled helicopter manual, Argus, Hemel Hempstead, Herts, England, 1981.
31. R. Hostetler, RAY's complete helicopter manual, R/C modeler corporation, 1991.

32. Arcamax Control Systems Inc., 150 E. Pleasant Hill Rd., Suite 174, Carbondale, IL 62901, Arcamax PI gyro.

33. S. Kim and D. Tilbury, Mathematical modeling and experimental identification of a model helicopter, in Proc of AIAA Guidance, Navigation, and Control Conf, 1998, pp. 203-213.

34. Polhemus Incorporated, P.O. Box 560, Colchester, VT 05446, 3SPACE User's Manual, December 1993.

35. L. Ljung, System identification: theory for the user, Prentice-Hall, Englewood Cliffs, NJ, 1999. 